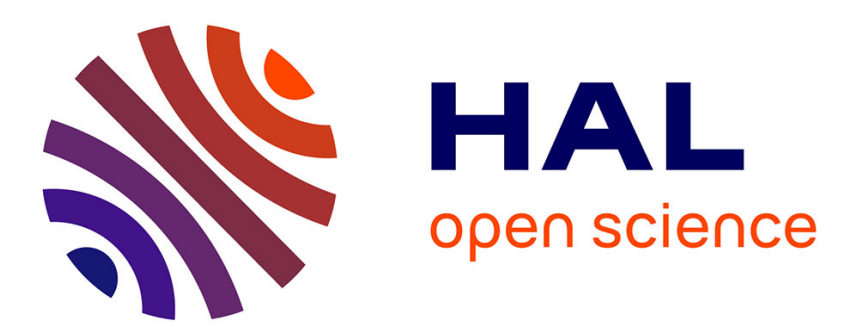

\title{
An asymptotically stable semi-lagrangian scheme in the quasi-neutral limit
}

Radoin Belaouar, Nicolas Crouseilles, Pierre Degond, Eric Sonnendrücker

\section{To cite this version:}

Radoin Belaouar, Nicolas Crouseilles, Pierre Degond, Eric Sonnendrücker. An asymptotically stable semi-lagrangian scheme in the quasi-neutral limit. Journal of Scientific Computing, 2009, 41, pp.341365. 10.1007/s10915-009-9302-4 . hal-00189383

\section{HAL Id: hal-00189383 https://hal.science/hal-00189383}

Submitted on 20 Nov 2007

HAL is a multi-disciplinary open access archive for the deposit and dissemination of scientific research documents, whether they are published or not. The documents may come from teaching and research institutions in France or abroad, or from public or private research centers.
L'archive ouverte pluridisciplinaire HAL, est destinée au dépôt et à la diffusion de documents scientifiques de niveau recherche, publiés ou non, émanant des établissements d'enseignement et de recherche français ou étrangers, des laboratoires publics ou privés. 


\title{
An asymptotically stable semi-lagrangian scheme in the quasi-neutral limit
}

\author{
R. Belaouar* N. Crouseilles ${ }^{\dagger} \quad$ P. Degond ${ }^{\ddagger} \quad$ E. Sonnendrücker ${ }^{\S}$
}

\begin{abstract}
This paper deals with the numerical simulations of the Vlasov-Poisson equation using a phase space grid in the quasi-neutral regime. In this limit, explicit numerical schemes suffer from numerical constraints related to the small Debye length and large plasma frequency. Here, we propose a semi-Lagrangian scheme for the Vlasov-Poisson model in the quasi-neutral limit. The main ingredient relies on a reformulation of the Poisson equation derived in [5] which enables asymptotically stable simulations. This scheme has a comparable numerical cost to that of an explicit scheme. Moreover, it is not constrained by a restriction on the size of the time and length step when the Debye length and plasma period go to zero. A stability analysis and numerical simulations confirm this.
\end{abstract}

\section{Contents}

1 Introduction $\quad 2$

2 The Vlasov-Poisson model and its quasi-neutral limit $\quad 4$

2.1 The Vlasov-Poisson system and its properties . . . . . . . . . . . . 4

2.2 The quasi-neutral model . . . . . . . . . . . . . . . . . . 6

2.3 The reformulated Poisson equation . . . . . . . . . . . 6

3 An asymptotic preserving scheme for the Vlasov-Poisson model $\quad 7$

3.1 The semi-Lagrangian method . . . . . . . . . . . . . 7

3.2 The classical time discretization for the Vlasov-Poisson model . . . . . . . 9

3.3 The asymptotically stable time discretization . . . . . . . . . . . . . 11

*INRIA Nancy-Grand-EST (CALVI Project). Université Louis Pasteur, 7 rue René Descartes 67084 Strasbourg cedex, France. belaouar@math.u-strasbg.fr

${ }^{\dagger}$ INRIA Nancy-Grand-EST (CALVI Project), and IRMA-Université Louis Pasteur. Université Louis Pasteur, 7 rue René Descartes 67084 Strasbourg cedex, France. crouseil@math.u-strasbg.fr

¥Institut de Mathématiques de Toulouse, Université de Toulouse et CNRS (UMR 5219). Université Paul Sabatier, 118, route de Narbonne, 31062 Toulouse cedex, France. degond@math.univ-toulouse.fr

$\S$ INRIA Nancy-Grand-Est (CALVI Project), and IRMA-Université Louis Pasteur. Université Louis Pasteur, 7 rue René Descartes 67084 Strasbourg cedex, France. sonnen@math.u-strasbg.fr 
4 Continuous dispersion relation of the linearized Vlasov-Poisson model 11

5 Stability analysis of the linearized equations $\quad 14$

5.1 Stability analysis of the linearized Vlasov-Ampère system . . . . . . . . . . 14

5.2 Stability analysis of the linearized Vlasov-RPE system . . . . . . . . . 16

6 Numerical results $\quad 18$

7 Conclusion $\quad 21$

8 Appendix: Details for the computation of the complex integrals $\quad 21$

\section{Introduction}

For many years, the modeling and numerical simulation of plasmas has been an active field of research. The description of the plasma is usually performed in two ways. On the one hand, fluid models which need that the system is close to a thermodynamical equilibrium to be valid. On the other side, kinetic models consider a phase space repartition of the particles, but numerical simulations are larger than fluid ones. Indeed, the high dimensionality of the kinetic models (6 dimensions plus the time) makes the simulations difficult to handle. However, when collisionless problems are studied, the use of kinetic models is necessary since fluid models cannot accurately describe the physics.

In addition, kinetic simulations are complex due to the large variety of scales involved in the system. Among them, there are two important physical length and time scales: the Debye length and the electron plasma period. The Debye length measures the typical length of charge unbalances whereas the electron plasma period characterizes the motion of the oscillations due to the electrostatic restoring forces when charge unbalances occur.

We are interested in this paper in the so-called quasi-neutral limit where both parameters are small compared with macroscopic lengths of interest. From a numerical point of view, a classical explicit scheme has to solve these micro-parameters in order to remain stable, which requires a very small time step and phase space cells. But on the other side, simulations have to be performed on macroscopic lengths, which makes kinetic simulations challenging.

Many asymptotic models have been derived to describe such regimes, but in situations where both quasi-neutral and non quasi-neutral regimes coexist, these models are not valid. Hence, hybrid approaches can be adopted (see [17, 21, 24]). However, a specific development is necessary to connect the models, and the interface has to be carefully described through an asymptotic analysis (see $[12,25]$ ) or thanks to physical considerations. Finally, these two points are quite difficult to handle numerically. Hence, it seems important to develop numerical methods which can handle in the two regimes.

The main goal of this work is to present Vlasov-type simulations (i.e. using a grid of the phase space) in the quasi-neutral regime. To that purpose, following the strategy introduced in $[5,6,9]$, a new numerical scheme is introduced, the stability analysis of 
which shows that its stability domain is independent of the Debye length. The present approach allows stable simulations even when the mesh does not resolve the Debye length and the plasma period.

As in [9], the Vlasov-Poisson model is studied with small values of the Debye length (which corresponds to the quasi-neutral regime). The Poisson equation is re-written in an equivalent form: the so-called Reformulated Poisson Equation (RPE). It has been first introduced in $[5,6]$ within the context of the fluid Euler-Poisson system, and the extension to the kinetic framework has been performed in [9]. The RPE enables to overcome the drastic reduction of time and space steps and is not more difficult or costly to solve numerically.

This work is based on the same model as [9] since the Vlasov equation is coupled with the RPE, but a semi-Lagrangian Vlasov solver is used in place of a Particle In Cell (PIC) solver. Such solvers are very often used for kinetic simulations (see [1, 18]) with the advantage that the computational cost of these methods remains acceptable, even in high dimensions. However, the inherent numerical noise becomes too significant for some applications. Hence, methods discretizing the Vlasov equation on a phase space grid have been proposed (see $[14,15,26]$ ). Unlike PIC methods, the distribution function is well resolved everywhere, even in zones where few plasma particles are present.

The semi-Lagrangian method can be viewed as an intermediate method between PIC methods and Eulerian methods. The mesh of the phase space is kept fixed in time (Eulerian method), and the Vlasov equation is integrated along the trajectories using the invariance of the distribution function along the characteristic curves (Lagrangian method). Interpolation is performed to evaluate the new value of the distribution function on the grid nodes. In particular, one of the advantages of such a method is to have a good description of the phase space (also in regions where the density is low), and unlike PIC methods, it is noiseless.

The main particularity of this work consists in the time integration of the trajectories and its coupling with the field solver. As in [9], the particle trajectories are computed using a semi-implicit symplectic integrator: the characteristics in velocity are integrated using an implicit electric potential evaluated at an explicit position. Semi-implicit time discretization of the characteristics has already been employed in $[4,20,22,23]$, but the use of the Reformulated Poisson Equation makes the approach different. This equation enables to predict a stable electric field even for small values of the Debye length $\lambda$. Moreover, the present approach does not suffer from unphysical decay of conserved quantities such as the total energy, which can prevent the asymptotic preserving property of the numerical scheme. Besides, as mentionned in $[5,6,9]$, the coupling with the RPE together with the new time integration has the same computational cost as the standard resolution of the Vlasov-Poisson equation.

Moreover, a stability analysis of the model is performed in the linear framework, proving that the numerical scheme is stable for small values of the Debye length $\lambda$, even if the time step does not resolve it. Such a study has been performed for the Euler-Poisson context in [11]. However, the strategy is different in the Vlasov case. Indeed, starting from the semi-discretized linearized version of the Vlasov equation coupled with the RPE, we 
derive a semi-discretized dispersion relation. The roots of this dispersion relation provide an indication on the stability of the numerical scheme. Indeed, when the imaginary part of the root is negative, then the numerical scheme is stable. As a comparison, we also derive a dispersion relation for the classical numerical scheme which does not enjoy such a property when the time step is bigger than the Debye length. This study emphasizes the Asymptotic Preserving property since the damping coefficient obtained by solving the dispersion relation presents the correct behavior as $\lambda$ goes to zero. These results are confirmed by the numerical results.

The paper is organized as follows. In the next part, we describe the Vlasov-Poisson model and introduce the Reformulated Poisson Equation. Then, we recall the main steps of the semi-Lagrangian method. Next, the asymptotically stable numerical scheme is presented with a classical scheme. A stability analysis is then performed on these two numerical schemes by solving the associated dispersion relation. Finally, some numerical results illustrate the efficiency of the new method compared to the classical one.

\section{The Vlasov-Poisson model and its quasi-neutral limit}

In this section, we present the Vlasov-Poisson system ans its quasi-neutral limit. As in [5], we show that the Poisson equation can be reformulated into an elliptic equation which does not degenerate in the quasi-neutral limit and, at the limit, provides an equation for the quasi-neutral potential.

\subsection{The Vlasov-Poisson system and its properties}

In this paper, we restrict ourselves to the one-dimensional Vlasov-Poisson system, even if this work straightforwardly extends to the multi-dimensional case.

Here, we consider only one species of particles, the electrons, and we assume that the ions form a uniform neutralizing background. Under these assuptions, the time evolution of the electron distribution function $f(t, x, v)$ in phase space $(x, v) \in \mathbb{R} \times \mathbb{R}$ (with $t$ the time, $x$ the spatial direction and $v$ the velocity) is given by the dimensionless Vlasov equation

$$
\partial_{t} f+v \partial_{x} f+\partial_{x} \phi \partial_{v} f=0,
$$

where the electric potential $\phi(t, x)$ is coupled to $f$ through the Poisson equation

$$
\lambda^{2} \partial_{x x} \phi(t, x)=\rho(t, x)-1, \quad \text { with } \quad \rho(t, x)=\int f(t, x, v) d v .
$$

In this one-dimensional context, this Poisson equation (2.2) is equivalent to the Ampère equation

$$
\partial_{t} E=\frac{j}{\lambda^{2}}, \quad j(t, x)=\int_{\mathbf{R}} v f(t, x, v) d v,
$$


where $E=-\partial_{x} \phi$ is the electric field.

Here the density $\rho$ has been normalized to the ion background density and the electron mass to unity. The dimensionless parameter $\lambda$ is the ratio of the Debye length to the length unit, or equivalently the ratio of the plasma period to the time unit. Here, velocities are normalized to ionic thermic velocity and space to a characteristic length of the problem.

In the sequel, we briefly recall some classical estimates on the Vlasov-Poisson system (2.1)-(2.2). First of all, mass and momentum are preserved with time,

$$
\frac{d}{d t} \int_{\mathbb{R} \times \mathbf{R}} f(t, x, v)\left(\begin{array}{c}
1 \\
v
\end{array}\right) d x d v=0, \quad t \in \mathbb{R}^{+} .
$$

Next, multiplying the Vlasov equation (2.1) by $|v|^{2}$ and performing an integration by parts, we find the conservation of the total energy $\mathcal{E}_{t}$ for the (2.1)-(2.2) system

$$
\frac{d \mathcal{E}_{t}}{d t}=\frac{d}{d t}\left(\mathcal{E}_{k}(t)+\mathcal{E}_{p}(t)\right)=0, \quad t \in \mathbb{R}^{+},
$$

where $\mathcal{E}_{k}$ denotes the kinetic energy and $\mathcal{E}_{p}$ the potential energy

$$
\mathcal{E}_{k}(t)=\int_{\mathbb{R} \times \mathbb{R}} f(t, x, v) \frac{|v|^{2}}{2} d x d v, \quad \mathcal{E}_{p}(t)=\frac{\lambda^{2}}{2} \int_{\mathbb{R}}\left|\partial_{x} \phi(t, x)\right|^{2} d x .
$$

On the other hand, we can define the characteristic curves of the Vlasov-Poisson equation (2.1)-(2.2) as the solutions of the following first order differential system

$$
\left\{\begin{array}{l}
\frac{d X}{d t}(t ; s, x, v)=V(t ; s, x, v) \\
\frac{d V}{d t}(t ; s, x, v)=\partial_{x} \phi(t, X(t ; s, x, v)),
\end{array}\right.
$$

with the initial conditions

$$
X(s ; s, x, v)=x, \quad V(s ; s, x, v)=v .
$$

We denote by $(X(t ; s, x, v), V(t ; s, x, v))$ the position in phase space at the time $t$, of a particle which was in $(x, v)$ at time $s$. Let say that $t \rightarrow(X(t ; s, x, v), V(t ; s, x, v))$ is the characteristic curves solution of (2.4). Then, the solution of the Vlasov-Poisson equation (2.1)-(2.2) is given by

$$
\begin{aligned}
f(t, x, v) & =f(s, X(s ; t, x, v), V(s ; t, x, v)) \\
& =f_{0}(X(0 ; t, x, v), V(0 ; t, x, v)), \quad(x, v) \in \mathbb{R} \times \mathbb{R}, \quad t \geq 0,
\end{aligned}
$$

where $f_{0}$ is a given initial condition of the Vlasov-Poisson equation. This equality means that the distribution function $f$ is constant along the characteristic curves which is the basis of the semi-Lagrangian method we recall in a next section. 


\subsection{The quasi-neutral model}

The quasi-neutral limit of the Vlasov-Poisson system $(\lambda \rightarrow 0)$ has been studied rigorously in a series of papers (for example see [2]).

Formally, passing to the limit $\lambda \rightarrow 0$ in (2.1)-(2.2) merely amounts to replacing the equation (2.2) by the quasi-neutrality constraint $\rho=1$. The Poisson equation is then lost, while the electrostatic potential becomes the Lagrange multiplier of the quasi-neutrality constraint. This is exactly the same in the incompressible Euler equations in which the pressure is a Lagrange multiplier for the divergence-free constraint.

Assuming that the quasineutrality constraint is satisfied initially, integrating (2.1) with respect to the velocity variable leads to the divergence-free constraint for the scaled electric current

$$
\partial_{x} \int v f d v=0 .
$$

Then, using (2.7) and after some computations that will be detailed in the next section, we obtain the following elliptic equation for the quasi-neutral potential $\phi$

$$
\partial_{x}^{2} \phi=\partial_{x}^{2} S,
$$

where $S$ is the second moment of the distribution function $f, S(t, x)=\int v^{2} f(t, x, v) d v$. In summary, the quasi-neutral model consists in the following system

$$
\begin{aligned}
& \frac{\partial f}{\partial t}+v \partial_{x} f+\partial_{x} \phi \partial_{v} f=0, \\
& \partial_{x}^{2} \phi=\partial_{x}^{2} S .
\end{aligned}
$$

We first note that the Vlasov-Poisson system (2.1)-(2.2) and (2.9)-(2.10) differ by the elliptic equations for the potential $\phi$ namely the Poisson equation (2.2) for the former and the quasi-neutral limit (2.10) for the latter.

A major difficulty is to find a direct way to obtain the equation (2.10) from the quasineutral limit of $(2.2)$. In $[5,6]$, in order to unify these two different equations, a new reformulation of the Poisson equation has been derived.

\subsection{The reformulated Poisson equation}

This present part recalls the main steps of the derivation of the Reformulated Poisson Equation (see $[5,6,9]$ ).

By taking the two first moments of the Vlasov equation, we get the continuity equation

$$
\partial_{t} \rho+\partial_{x} j=0,
$$

and the equation evolving the current density $j$

$$
\partial_{t} j+\partial_{x} S-\rho \partial_{x} \phi=0,
$$


where $\rho=\int f(v) d v, j=\int v f(v) d v$ and $S=\int v^{2} f(v) d v$. In order to eliminate the current $j$, we make the difference between the time derivative of (2.11) and the divergence of (2.12). It follows

$$
\partial_{t t} \rho-\partial_{x x} S+\partial_{x}\left(\rho \partial_{x} \phi\right)=0 .
$$

Now, using the Poisson (2.2) to replace $\rho$ in the first term of (2.13) gives the Reformulated Poisson Equation

$$
-\partial_{x}\left[\left(\lambda^{2} \partial_{t t}+\rho\right) \partial_{x} \phi\right]=-\partial_{x x} S
$$

which is equivalent to the original one if initially the Poisson equation (2.2) and its time derivative are satisfied.

In the quasi-neutral limit $(\lambda \rightarrow 0)$, the reformulated equation (2.14) formally converges toward the quasi-neutral potential elliptic equation (2.10). It does not degenerate into an algebraic equation like the Poisson equation (2.2) does. Then the reformulated system

$$
\begin{aligned}
& \frac{\partial f}{\partial t}+v \partial_{x} f+\partial_{x} \phi \partial_{v} f=0, \\
& -\partial_{x}\left[\left(\lambda^{2} \partial_{t t}+\rho\right) \partial_{x} \phi\right]=-\partial_{x x} S
\end{aligned}
$$

seems to be an appropriate framework to deal with problems which are partly or totally in the quasi-neutral regime.

In the next section, we show how we can use this reformulated system to derive an asymptotic time strategy for the Vlasov-Poisson problem.

\section{An asymptotic preserving scheme for the Vlasov- Poisson model}

In this part, we describe a numerical scheme used to solve the Vlasov-Poisson system. In a previous work of P. Degond et.al [9], a PIC method was used to solve the Vlasov-Poisson equation. Although they could deal with unresolved Debye length and plasma electron period and get stable simulations, they observed an unphysical strong decay of the total energy which could not permit to verify if the PIC method enjoys the asymptotic preserving property. In this work, we propose to use a semi-Lagrangian method to overcome this lack of energy conservation.

\subsection{The semi-Lagrangian method}

In this section, we will recall the principles of the semi-Lagrangian method for the VlasovPoisson equation (see [26] for more details) in two dimensions of the phase space.

First of all, we introduce the finite set of mesh points $\left(x_{i}, v_{j}\right), i=0, \ldots, N_{x}$ and $j=$ $0, \ldots, N_{v}$ to discretize the phase space computational domain. Then, given the value of 
the distribution function $f$ at the mesh points at any given time step $t^{n}$, we obtain the new value at mesh points $\left(x_{i}, v_{j}\right)$ at $t^{n+1}$ using

$$
f\left(t^{n}+\Delta t, x_{i}, v_{j}\right)=f\left(t^{n}, X^{n}, V^{n}\right),
$$

where the notations $\left(X^{n}, V^{n}\right)=X\left(t^{n} ; t^{n}+\Delta t, x_{i}, v_{j}\right), V\left(t^{n} ; t^{n}+\Delta t, x_{i}, v_{j}\right)$ are used for the solutions of (2.4), and $\Delta t$ stands for the time step. For each mesh point $\left(x_{i}, v_{j}\right)$, the distribution function $f$ is then computed at $t^{n+1}$ by the two following steps

1. Find the starting point of the characteristic ending at $\left(x_{i}, v_{j}\right)$, which is $X^{n}$ and $V^{n}$.

2. Compute $f\left(t^{n}, X^{n}, V^{n}\right)$ by interpolation, $f$ being known only at mesh points at time $t^{n}$.

Now, for the general case, in order to deal with step 1, we need to introduce a time discretization of (2.4). A lot of numerical methods exist for the resolution of the characteristic curves, given by the following ordinary differential equations

$$
\begin{aligned}
& \frac{d X}{d t}=V \\
& \frac{d V}{d t}=\partial_{x} \phi(t, X) .
\end{aligned}
$$

Here, we want to use a robust and stable scheme which can take into account the spatial and time oscillations of the electric potential $\phi$ when the parameter $\lambda$ tends towards 0 . To reach this goal, the first difficulty is related to the time discretization of $\phi$ in the right hand side of (3.2). In the context where the plasma is at equilibrium, it refers to a source term in the momentum conservation's law of the Euler equations. In the work of S. Fabre (see [13]), it is proven that a necessary condition for stability for the Euler-Poisson system is the use of an implicit time discretization of the advection term $\partial_{x} \phi$. We therefore do the same for the time discretization of $\phi$ in (3.2).

The second difficulty is related to the time discretization of (3.1) coupled to the space discretization of the right hand side of (3.2). In order to preserve the total mass quantity for all time and to preserve the areas of the transformation $\left(X^{n}, V^{n}\right) \rightarrow\left(X^{n+1}, V^{n+1}\right)$, we have to use the well known Euler symplectic schemes for (3.1)-(3.2) (see [27] for more details).

Then, we have two possible alternatives to discretize (3.1)-(3.2). The first one we call (EI) is (E for explicit in space and I for implitit in velocity)

$$
\begin{aligned}
& \frac{X^{n+1}-X^{n}}{\Delta t}=V^{n}, \\
& \frac{V^{n+1}-V^{n}}{\Delta t}=\partial_{x} \phi^{n+1}\left(X^{n+1}\right),
\end{aligned}
$$


and the second one (IE) writes

$$
\begin{aligned}
& \frac{X^{n+1}-X^{n}}{\Delta t}=V^{n+1}, \\
& \frac{V^{n+1}-V^{n}}{\Delta t}=\partial_{x} \phi^{n+1}\left(X^{n}\right) .
\end{aligned}
$$

But some basic computations on the $(E I)$ scheme lead to

$$
\frac{X^{n+1}-2 X^{n}+X^{n-1}}{\Delta t^{2}}=\partial_{x} \phi^{n}\left(X^{n}\right),
$$

whereas the same ones for the $(I E)$ scheme give

$$
\frac{X^{n+1}-2 X^{n}+X^{n-1}}{\Delta t^{2}}=\partial_{x} \phi^{n+1}\left(X^{n}\right) .
$$

The two schemes (3.7) and (3.8) correspond to an explicit and an implicit time discretization of the equation describing the motion of electrons

$$
\frac{d^{2} X}{d t^{2}}=\partial_{x} \phi(t, X) .
$$

In order to get stable numerical results with respect to the time step $\Delta t$ and the parameter $\lambda$ and since we deal with strong oscillations in space and in time of the electric potentiel $\phi$, we have to choose the symplectic scheme (IE) to solve (3.1)-(3.2).

Therefore the starting point of the characteristic curves ending at $\left(X^{n+1}, V^{n+1}\right)$ is computed thanks to the following numerical scheme

$$
\begin{aligned}
& X^{n}=X^{n+1}-\Delta t V^{n+1}, \\
& V^{n}=V^{n+1}-\Delta t \partial_{x} \phi^{n+1}\left(X^{n}\right) .
\end{aligned}
$$

The second step of the semi-Lagrangian method deals with the interpolation of $f^{n}\left(X^{n}, V^{n}\right)$ by using the values of $f^{n}$ on the mesh points. This is done by using local cubic B-splines. For more details on this step, we refer the reader to [8].

\subsection{The classical time discretization for the Vlasov-Poisson model}

In this subsection, the Ampère equation will be used to predict the electric field at time $t^{n+1}\left(E^{n+1}=-\partial_{x} \phi^{n+1}\right)$ in (3.10). Indeed, we use the fact that in one dimension of space, the Poisson equation (2.2) and the Ampère equation (2.3) are equivalent (note that the methodology can be extended to multidimensional problems using the continuity equation, see [7] for example). In the rest of the paper, the use of the Ampère equation in order to predict the electric field at time $t^{n+1}$ will be referred to the "classical time discretization". Its time discretization writes

$$
E_{i}^{n+1}=E_{i}^{n}+\frac{\Delta t}{\lambda^{2}} j_{i}^{n},
$$


where $\Delta t$ is the time step, $E_{i}^{n}$ is the electric field evaluated at $t=t^{n}$ in $x=x_{i}$. Finally, $j_{i}^{n}$ denotes the current evaluated at time $t^{n}$ in $x_{i}$, and is given by

$$
j_{i}^{n}=\sum_{j=0}^{N_{v}} f\left(t^{n}, x_{i}, v_{j}\right) v_{j} \Delta v,
$$

with $\Delta v$ the velocity step.

Hence the classical numerical scheme can be decomposed into the following steps.

Let us suppose that $f\left(t^{n}, x_{i}, v_{j}\right),\left(\partial_{x} \phi^{n}\right)_{i}$ are known on the mesh points

Step 1. Computation of a prediction of $E_{i}^{n+1}$, called $\tilde{E}_{i}^{n+1}$, by solving the Ampère equation

$$
\tilde{E}_{i}^{n+1}=E_{i}^{n}+\frac{\Delta t}{\lambda^{2}} j_{i}^{n}
$$

where $j_{i}^{n}$ is computed via (3.12).

Step 2. Resolution of (2.4)

- Backward advection of $\Delta t$ in the spatial direction

$$
X^{n}=X^{n+1}-\Delta t V^{n+1} .
$$

- Backward advection of $\Delta t$ in the velocity direction

$$
V^{n}=V^{n+1}-\Delta t \partial_{x} \phi^{n+1}\left(X^{n}\right),
$$

Step 3. Interpolation of $f\left(t^{n}, X^{n}, V^{n}\right)$ and updating of the distribution function thanks to the following equality

$$
f\left(t^{n+1}, X^{n+1}, V^{n+1}\right)=\Pi f\left(t^{n}, X^{n}, V^{n}\right),
$$

where $\Pi$ is an interpolation operator.

Step 4. Computation of the density $\rho^{n+1}\left(X^{n+1}\right)$

$$
\rho^{n+1}\left(X^{n+1}\right)=\int_{\mathbf{R}} f\left(t^{n+1}, X^{n+1}, v\right) d v,
$$

and resolution of the Poisson equation at time $t^{n+1}$ to get $\phi^{n+1}$ and $E^{n+1}$.

It is well known that the stability of this classical scheme requires a space and a time step which resolve the parameter $\lambda$ (the numerical results will show this fact). But this classical approach will be used as a reference to make comparison with the new approach. 


\subsection{The asymptotically stable time discretization}

As evoked previously, we use the Reformulated Poisson Equation (2.14) to compute the electric potential at time $t^{n+1}$. To that purpose, a time discretization has to be performed, deduced from a time discretization of the Euler-Poisson equation (see [5, 6, 9]).

In the sequel, we fastly recall the main steps allowing to derive a time disretization of the Reformulated Poisson Equation. The starting point is the semi-discretization in time of (2.11)-(2.12) in the following way

$$
\begin{aligned}
& \frac{\rho^{k+1}-\rho^{k}}{\Delta t}+\partial_{x} j^{k+1}=0, \\
& \frac{j^{k+1}-j^{k}}{\Delta t}+\partial_{x} S^{k}-\rho^{k} \partial_{x} \phi^{k+1}=0 .
\end{aligned}
$$

Now, we perform the same computations as in the continuous case (see section 2.3): we take the discrete time difference of (3.13) and we combine it with the space derivative of (3.14) to eliminate the discrete moment $j^{k}$. This leads to

$$
\frac{\rho^{k+1}-2 \rho^{k}+\rho^{k-1}}{\Delta t^{2}}+\partial_{x}\left(\rho^{k} \partial_{x} \phi^{k+1}\right)=\partial_{x}^{2} S^{k} .
$$

By substituting the density $\rho^{k+1}$ by $\left(1+\lambda^{2} \partial_{x}^{2} \phi^{k+1}\right)$ thanks to the Poisson equation which we suppose satisfied at time $t^{n+1}$, we get the semi-implicit time differencing of $(2.14)$

$$
-\partial_{x}\left(\left(\rho^{k} \Delta t^{2}+\lambda^{2}\right) \partial_{x} \phi^{k+1}\right)=-\Delta t^{2} \partial_{x}^{2} S^{k}-2 \rho^{k}+\rho^{k-1}+1 .
$$

Let us remark that (3.16) is an elliptic problem which allows to compute $\phi^{k+1}$ thanks to quantities at time $t^{n}$ and which does not degenerate when $\lambda$ goes to zero; moreover, its numerical resolution has the same cost as the traditional Poisson equation.

The spatial approximation of (3.16) is performed in a usual way, by discretizing the space derivatives on the fixed grid $\left(x_{i}\right)_{i}$ using uncentered finite differences. The reader is refered to [6] for more details.

\section{Continuous dispersion relation of the linearized Vlasov- Poisson model}

In this section, we study the dispersion relation of the linearized Vlasov-Poisson model for different values of $\lambda$. To derive the dispersion relation; the Vlasov-Poisson model (2.1)-(2.2) is linearized around a equilibrium Maxwellian distribution function

$$
f_{0}(x, v)=\frac{1}{\sqrt{2 \pi}} \exp \left(-\frac{v^{2}}{2}\right), E_{0}(x)=0 .
$$

We may reformulate the Vlasov-Poisson system (2.1)-(2.2) as equations for the perturbations $f_{1}$ and $E_{1}$ of the equilibrium (4.1) so that

$$
f=f_{0}+f_{1}, \quad E=0+E_{1} .
$$


We deduce that they satisfy the linearized Vlasov-Poisson equation

$$
\begin{aligned}
& \partial_{t} f_{1}+v \partial_{x} f_{1}-E_{1} \partial_{v} f_{0}=0, \\
& \lambda^{2} \partial_{x} E_{1}=-\int f_{1} d v .
\end{aligned}
$$

Note that the linearized Poisson equation is equivalent to

$$
\lambda^{2} \partial_{t} E_{1}=\int v f_{1} d v
$$

which corresponds to the linearization of the Ampère equation around the Maxwellian steady-state.

The dispersion relation of (4.2)-(4.3) (see[10]) is

$$
D(\omega, \xi, \lambda)=1+\frac{1}{\lambda^{2} \xi^{2}} \int \frac{\partial_{v} f_{0}}{\frac{\omega}{\xi}-v} d v .
$$

As in [16], this function $D(4.5)$ can be reformulated as

$$
D(\omega, \xi, \lambda)=1+\frac{1}{\lambda^{2} \xi^{2}}\left(1+\sqrt{\frac{\pi}{2}} \frac{\omega}{\xi} \exp \left(-\frac{\omega^{2}}{2 \xi^{2}}\right)\left(i-\operatorname{erfi}\left(\frac{\omega}{\sqrt{2} \xi}\right)\right)\right) .
$$

where erfi is the imaginary error function defined such that

$$
\operatorname{erfi}(0)=0, \text { and } \frac{d}{d x} \operatorname{erfi}(x)=\frac{2}{\sqrt{\pi}} \exp \left(x^{2}\right) .
$$

For reading convenients, the details of the computations from (4.5) to (4.6) have been put in Appendix.

This last formulation enables to compute numerically $\omega$ as a function of $(\xi, \lambda)$. In the sequel, we plot the imaginary part of the solutions of (4.6) as a function of $\xi$ for different values of $\lambda$.

We can observe that there exists at least two curves of solutions of (4.6). We plot on Figs. 1, 2 two curves of solutions of the dispersion relation: the absolute value of the Imaginary part of the solution $\omega=\omega_{r}+i \omega_{i}$ is plotted as a function of the wave number, for different values of $\lambda$. Several curves of solutions exist, but we restrict ourselves to solutions with small $\omega_{i}$.

In the literature, numerical simulations capture the wave associated with the smallest $\omega_{i}$ since the others waves are damped very fastly; however, residual of these highly damped waves can be observed at the beginning of the simulations: the first oscillation is usually larger than the following ones (see $[7,14]$ ). 


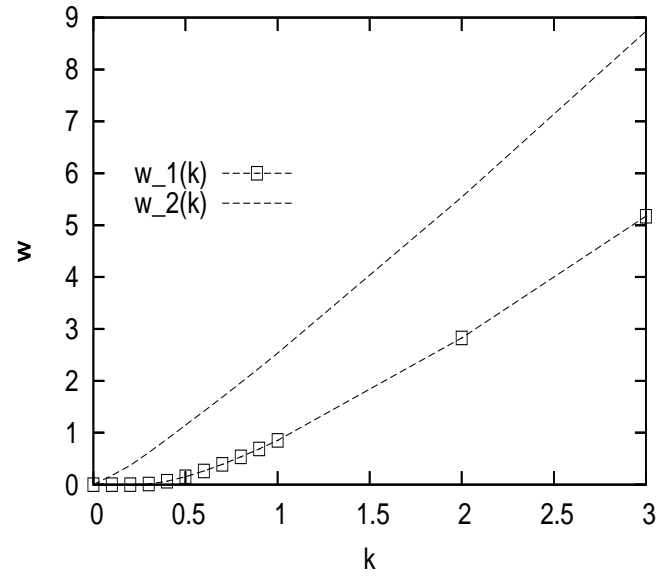

(a)

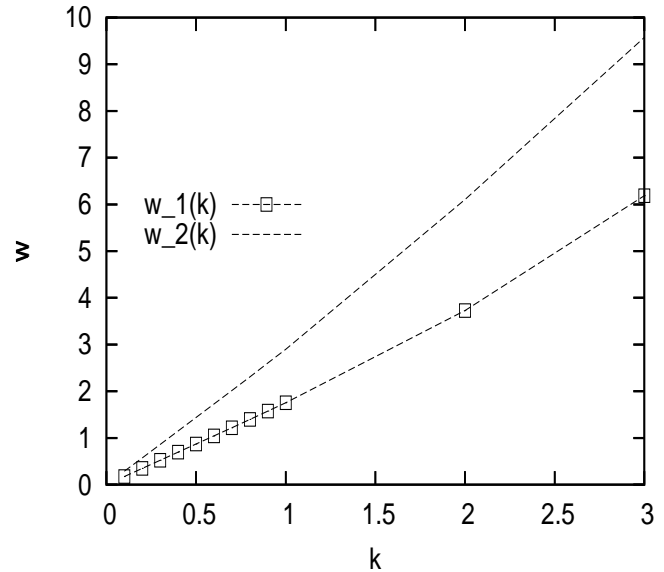

(b)

Figure 1: Absolute value of the Imaginary part of the solution to (4.6) as a function of $\xi$ : (a) $\lambda=1$, (b) $\lambda=10^{-1}$.

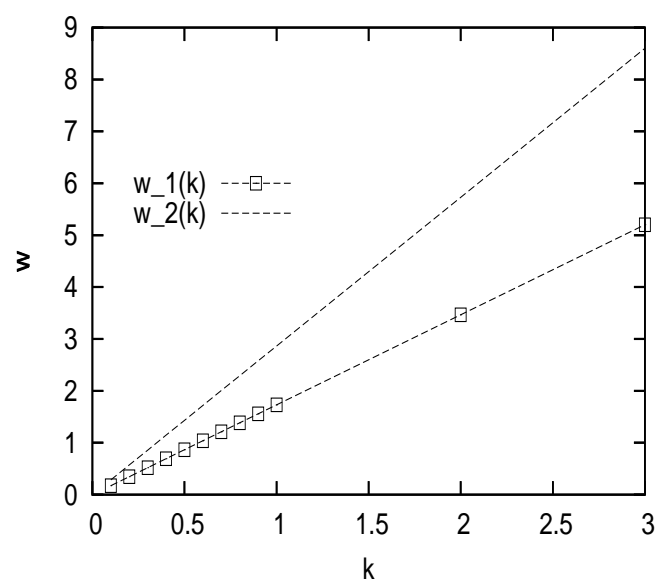

(a)

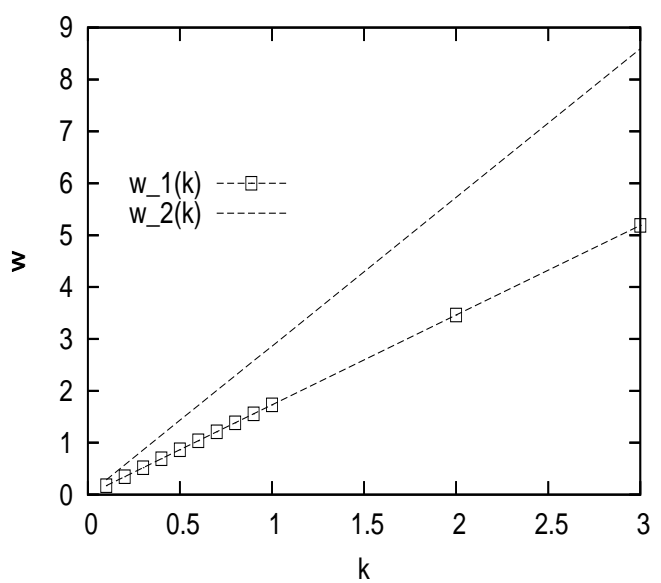

(b)

Figure 2: Absolute value of the Imaginary part of the solution to (4.6) as a function of $\xi$ : (a) $\lambda=10^{-2}$, (b) $\lambda=10^{-3}$. 


\section{$5 \quad$ Stability analysis of the linearized equations}

In this section, we study the linear stability of a semi-discretization in time of the VlasovPoisson (Vlasov-Ampère) system and of the Vlasov-RPE system. For each system, we start from the time discretization of its linearized version. Then, by using a spatial Fourier transform, the discrete dispersion relation is computed for each scheme, which enables to study the stability of the time discretization. Even if looking at the stability of a linearized semi-discretized version of the initial model is quite restrictive, this study is easier and can give some indications of the behavior of the fully discretized model. Let us mention [11], in which the authors perform an similar study for the Euler-Poisson and Euler-RPE systems; asymptotic stability is then proved when the RPE is used.

\subsection{Stability analysis of the linearized Vlasov-Ampère system}

In order to analyse the numerical stability of the semi-discrete scheme, we start from the time discretization of the linearized version the Vlasov-Poisson model (4.2)(4.4)

$$
\begin{aligned}
& \frac{f_{1}^{n+1}-f_{1}^{n}}{\Delta t}+v \partial_{x} f_{1}^{n+1}-E_{1}^{n+1} \partial_{v} f_{0}=0, \\
& \frac{\lambda^{2}}{\Delta t}\left(E_{1}^{n+1}-E_{1}^{n}\right)=\int v f_{1}^{n} d v,
\end{aligned}
$$

where the flux term as well as the electric field as considered implicit, following [11]. In order to analyse the stability of the numerical scheme (5.1)-(5.2), it is customary, at this point, to introduce the Fourier transforms in space of the perturbed distribution function and of the electric field. The numerical scheme in Fourier space reads

$$
\begin{aligned}
& \frac{\widehat{f}_{1}^{n+1}-\widehat{f}_{1}^{n}}{\Delta t}+i \xi v \widehat{f}_{1}^{n+1}-\widehat{E}_{1}^{n+1} \partial_{v} f_{0}=0, \\
& \frac{\lambda^{2}}{\Delta t}\left(\widehat{E}_{1}^{n+1}-\widehat{E}_{1}^{n}\right)=\int v \widehat{f}_{1}^{n} d v
\end{aligned}
$$

where $\widehat{f}^{n}, \widehat{E}^{n}$ denote the spatial Fourier transform of $f^{n}, E^{n}$ respectively.

Let us follow the standard procedure for analyzing small amplitudes waves. Assuming that all perturbed quantities evolve in time like $\exp (-i \omega t)$, the Fourier transforms in space of $\widehat{f}^{n}$ and $\widehat{E}^{n}$ can be written as

$$
\widehat{f}_{1}^{n}=C_{f} \exp (-i \omega n \Delta t), \widehat{E}^{n}=C_{e} \exp (-i \omega n \Delta t)
$$

where $C_{f}$ and $C_{e}$ are functions of $\xi$. Seeking the solution of (5.3)-(5.4) under the form (5.5) leads to

$$
\begin{aligned}
& C_{f}[\exp (-i \omega \Delta t)(1+i \Delta t \xi v)-1]=C_{e} \Delta t \exp (-i \omega \Delta t) \partial_{v} f_{0} \\
& C_{e} \frac{\lambda^{2}}{\Delta t}(\exp (-i \omega \Delta t)-1)=\int v C_{f} d v
\end{aligned}
$$


which gives

$$
\begin{gathered}
C_{f}=\frac{C_{e} \Delta t \exp (-i \omega \Delta t) \partial_{v} f_{0}}{\exp (-i \omega \Delta t)(1+i \Delta t \xi v)-1}, \\
C_{e} \frac{\lambda^{2}}{\Delta t}(\exp (-i \omega \Delta t)-1)=\int v C_{f} d v .
\end{gathered}
$$

Since we deal with non-zero solutions, plugging the expression of $C_{f}$ given by (5.6) in (5.7) gives

$\frac{\lambda^{2}}{\Delta t}(\exp (-i \omega \Delta t)-1)-\Delta t \exp (-i \omega \Delta t) \int \frac{v \partial_{v} f_{0}}{\exp (-i \omega \Delta t)-1+i \exp (-i \omega \Delta t) \Delta t \xi v)} d v=0$

which can be rewritten as

$$
\frac{\lambda^{2}}{\Delta t}(\exp (-i \omega \Delta t)-1)-\frac{i}{\xi} \int \frac{v \partial_{v} f_{0}}{\frac{\exp (i \omega \Delta t)-1}{i \Delta t \xi}-v} d v=0 .
$$

Some basic computations lead to

$$
1-i \frac{\Delta t}{\lambda^{2} \xi(\exp (-i \omega \Delta t)-1)} \int \frac{v \partial_{v} f_{0}}{\widetilde{a}-v} d v=0,
$$

with $\widetilde{a}=\frac{\exp (i \omega \Delta t)-1}{i \Delta t \xi}=\frac{a}{\xi}$. Since the following equality holds

$$
\int \frac{v \partial_{v} f_{0}}{\widetilde{a}-v} d v=\widetilde{a} \int \frac{\partial_{v} f_{0}}{\widetilde{a}-v} d v
$$

the discrete dispersion relation associated to the Vlasov-Ampère discretization is given by

$$
D_{1}^{\Delta t}(\omega, \xi, \lambda)=1+\frac{\exp (i \omega \Delta t)}{\lambda^{2} \xi^{2}} \int \frac{\partial_{v} f_{0}}{\widetilde{a}-v} d v
$$

Moreover, for all $\alpha \in \mathbb{C}$, we have (see the Appendix for more details)

$$
\int \frac{\partial_{v} f_{0}}{\alpha-v} d v=1+\alpha \sqrt{\frac{\pi}{2}} \exp \left(-\alpha^{2} / 2\right)(i-\operatorname{erfi}(\alpha / \sqrt{2})) .
$$

This previous computations finally give the following discrete dispersion relation

$$
D_{1}^{\Delta t}=1+\frac{\exp (i \omega \Delta t)}{\lambda^{2} \xi^{2}}\left(1+\sqrt{\frac{\pi}{2}} \frac{a}{\xi} \exp \left(-\frac{a^{2}}{2 \xi^{2}}\right)\left(i \operatorname{sign}(\xi)-\operatorname{erfi}\left(\frac{a}{\sqrt{2} \xi}\right)\right)\right),
$$

with $a=(\exp (i \omega \Delta t)-1) /(i \Delta t)$. Remark that, since $\lim _{\Delta t \rightarrow 0} a=\omega$, in the limit $\Delta t$ tends towards 0 , we recover the continuous dispersion relation (4.5)

$$
\lim _{\Delta t \rightarrow 0} D_{1}^{\Delta t}(\omega, \xi, \lambda)=D(\omega, \xi, \lambda)
$$


Thanks to this formulation of the dispersion relation (5.10), we are able to compute $\omega$ as a function of $(\xi, \lambda, \Delta t)$. The main goal consists in the determination of the behavior of the small amplitudes perturbed waves: if $\operatorname{Im}(\omega)<0$, the perturbations are damped and the numerical scheme is stable whereas if $\operatorname{Im}(\omega)>0$, the numerical scheme is then unstable. The numerical results are resumed in the table 1 . We can observe that the stability condition has to be respected; indeed when $\Delta t>\lambda$, we find $\operatorname{Im}(\omega)>0$ and the numerical scheme is then unstable.

\begin{tabular}{|c||c||c||c||c||c|}
\hline$\Delta t, \lambda$ & 1 & $10^{-1}$ & $10^{-2}$ & $10^{-3}$ & $10^{-4}$ \\
\hline \hline $10^{-1}$ & -0.8808 & -0.1506 & +45.55 & +91.8 & +137.86 \\
\hline $10^{-2}$ & -0.8563 & -1.8028 & -1.7806 & +1381 & +1842 \\
\hline $10^{-3}$ & -0.8518 & -1.7585 & -1.7377 & -1.7376 & +18420 \\
\hline $10^{-4}$ & -0.8513 & -1.7538 & -1.7533 & -1.7331 & -1.7333 \\
\hline 0 & -0.8513 & -1.7533 & -1.7528 & -1.7326 & -1.7326 \\
\hline
\end{tabular}

Table 1: Imaginary part of the root of the dispersion relation associated to the VlasovAmpère model in the implicit case: $\operatorname{Im}(\omega)$ for $\xi=1$ as a function of $(\Delta t, \lambda)$.

\subsection{Stability analysis of the linearized Vlasov-RPE system}

In this part, we perform the same analysis as previously for the Vlasov-RPE system

$$
\begin{aligned}
& \frac{\partial f}{\partial t}+v \partial_{x} f-E \partial_{v} f=0, \\
& -\partial_{x}\left[\left(\lambda^{2} \partial_{t t}+\rho\right) E\right]=\partial_{x x} S .
\end{aligned}
$$

The linearized Vlasov-RPE system around the Maxwellian steady state writes

$$
\begin{aligned}
& \partial_{t} f_{1}+v \partial_{x} f_{1}-E_{1} \partial_{v} f_{0}=0, \\
& \partial_{x}\left(\lambda^{2} \partial_{t}^{2} E_{1}+E_{1}\right)=-\partial_{x}^{2} S_{1},
\end{aligned}
$$

with $S_{1}(t, x)=\int v^{2} f_{1}(t, x, v) d v$.

In order to recover the continuous dispersion relation which permits to analyse the small amplitudes waves, we assume that all perturbed quantities vary with $(x, t)$ like $\exp (i(\xi x-\omega t))$. Thus equations (5.13)-(5.14) reduce to

$$
\begin{aligned}
& i(\omega-\xi v) C_{f}+C_{e} \partial_{v} f_{0}=0, \\
& i \xi\left(1-\omega^{2} \lambda^{2}\right) C_{e}=\xi^{2} \int v^{2} C_{f} d v
\end{aligned}
$$

respectively. Solving the first of these equations for $C_{f}$ and substituting into the integral in the second, we formally get, (if $C_{e}$ is non-zero) the following dispersion relation

$$
\widetilde{D}=\frac{1}{\xi}-\frac{\omega^{2} \lambda^{2}}{\xi}+\int \frac{v^{2} \partial_{v} f_{0}}{v \xi-\omega} d v=0 .
$$


Using the fact that

$$
\int \frac{v^{2} \partial_{v} f_{0}}{v \xi-\omega} d v=-\frac{1}{\xi}+\frac{\omega^{2}}{\xi^{2}} \int \frac{\partial_{v} f_{0}}{v \xi-\omega} d v
$$

we get

$$
\widetilde{D}(\omega, \xi, \lambda)=\frac{\omega^{2} \lambda^{2}}{\xi}\left(1+\frac{1}{\lambda^{2} \xi^{2}} \int \frac{\partial_{v} f_{0}}{\frac{\omega}{\xi}-v} d v\right)=0
$$

which is the same dispersion relation as for the linearized Vlasov-Poisson equation multiplied by $(\omega \lambda / \xi)^{2}$.

We compute the time approximate solutions of the linearized Vlasov-RPE system (5.13)-(5.14) with the following numerical scheme

$$
\begin{aligned}
& \frac{f^{n+1}-f^{n}}{\Delta t}+v \partial_{x} f^{n+1}-E^{n+1} \partial_{v} f_{0}=0, \\
& \lambda^{2} \frac{\partial_{x} E^{n+1}-2 \partial_{x} E^{n}+\partial_{x} E^{n-1}}{\Delta t^{2}}+\partial_{x} E^{n+1}=-\partial_{x}^{2} \int v^{2} f^{n} d v,
\end{aligned}
$$

The stability analysis is done using the space Fourier transform of (5.18)-(5.19)

$$
\begin{aligned}
& \frac{\widehat{f}^{n+1}-\widehat{f}^{n}}{\Delta t}+i \xi v \widehat{f}^{n+1}-\widehat{E}^{n+1} \partial_{v} f_{0}=0, \\
& i \frac{\lambda^{2}}{\Delta t^{2}}\left(\widehat{E}^{n+1}-2 \widehat{E}^{n}+\widehat{E}^{n-1}\right)+i \widehat{E}^{n+1}=\xi \int v^{2} \widehat{f}^{n} d v .
\end{aligned}
$$

Note that the equation (5.21) is still valid when $\xi=0$.

As in the Vlasov-Ampère case, we use the decomposition (5.5) for the linear stability analysis.

Seeking the solution of (5.20)-(5.21) under the form (5.5) leads to

$$
\begin{aligned}
& C_{f}[\exp (-i \omega \Delta t)(1+i \Delta t \xi v)-1]=C_{e} \Delta t \exp (-i \omega \Delta t) \partial_{v} f_{0} \\
& i \frac{\lambda^{2}}{\Delta t}(\exp (-i \omega \Delta t)+\exp (i \omega \Delta t)-2)+i \exp (-i \omega \Delta t) C_{e}=\xi \int v^{2} C_{f} d v
\end{aligned}
$$

Since we deal with non-zero solutions, plugging the expression of $C_{f}$ given by $(5.22)$ in (5.23) leads to

$$
i \frac{2 \lambda^{2}}{\Delta t^{2}}(\cos (\omega \Delta t)-1)+i \exp (-i \omega \Delta t)=\frac{\xi}{C_{e}} \int v^{2} C_{f} d v
$$

Using the fact that

$$
\int v^{2} C_{f} d v=\frac{i C_{e}}{\xi} \int \frac{v^{2} \partial_{v} f_{0}}{\widetilde{a}-v} d v, \text { with } \tilde{a}=\frac{\exp (-i \omega \Delta t)-1}{i \Delta t \xi},
$$


we get

$$
-i \frac{4 \lambda^{2}}{\Delta t^{2}} \sin ^{2}\left(\frac{\omega \Delta t}{2}\right)+i \exp (-i \omega \Delta t)=i \int \frac{v^{2} \partial_{v} f_{0}}{\widetilde{a}-v} d v
$$

Since the following equality holds

$$
\int \frac{v^{2} \partial_{v} f_{0}}{\widetilde{a}-v} d v=1+\widetilde{a}^{2} \int \frac{\partial_{v} f_{0}}{\widetilde{a}-v} d v
$$

the discrete dispersion relation associated to the Vlasov-RPE discretization (5.20)-(5.21) is

$$
D_{2}^{\Delta t}(\omega, \xi, \lambda)=1-\exp (-i \omega \Delta t)+\frac{4 \lambda^{2}}{\Delta t^{2}} \sin ^{2}\left(\frac{\omega \Delta t}{2}\right)+\widetilde{a}^{2} \int \frac{\partial_{v} f_{0}}{\widetilde{a}-v} d v .
$$

The previous computations give the following discrete dispersion relation

$$
\begin{aligned}
D_{2}^{\Delta t} & =1-\exp (-i \omega \Delta t)+\frac{4 \lambda^{2}}{\Delta t^{2}} \sin ^{2}\left(\frac{\omega \Delta t}{2}\right) \\
& +\frac{a}{\xi}\left(1+\sqrt{\frac{\pi}{2}} \frac{a}{\xi} \exp \left(-\frac{a^{2}}{2 \xi^{2}}\right)\left(i \operatorname{sign}(\xi)-\operatorname{erfi}\left(\frac{a}{\sqrt{2} \xi}\right)\right)\right)
\end{aligned}
$$

Remark that, in the limit $\Delta t$ tends towards 0 , we recover the continuous dispersion relation $(5.17)$

$$
\lim _{\Delta t \rightarrow 0} D_{2}^{\Delta t}(\omega, \xi, \lambda)=\xi \widetilde{D}(\omega, \xi, \lambda) .
$$

The numerical solutions of the dispersion relation $D_{2}^{\Delta t}$ are exposed in the tabular 2 where the imaginary part of $\omega$ is written as a function of $\Delta t$ and for different values of $\lambda$. As expected, the numerical scheme is stable for all values of $\lambda$ and $\Delta t$ since all the values of the imaginary part of $\omega$ are negative.

\begin{tabular}{|c||c||c||c||c||c|}
\hline$\Delta t, \lambda$ & 1 & $10^{-1}$ & $10^{-2}$ & $10^{-3}$ & $10^{-4}$ \\
\hline \hline $10^{-1}$ & -0.8949 & -2.0081 & -1.9817 & -1.9817 & -1.9817 \\
\hline $10^{-2}$ & -0.8573 & -1.7924 & -1.7708 & -1.7707 & -1.7707 \\
\hline $10^{-3}$ & -0.8519 & -1.7574 & -1.7367 & -1.7366 & -1.7366 \\
\hline $10^{-4}$ & -0.8514 & -1.7537 & -1.7332 & -1.7330 & -1.7330 \\
\hline 0 & -0.8513 & -1.7533 & -1.7328 & -1.7326 & -1.7326 \\
\hline
\end{tabular}

Table 2: Imaginary part of the root of the dispersion relation associated to the VlasovRPE model in the implicit case: $\operatorname{Im}(\omega)$ for $\xi=1$ as a function of $(\Delta t, \lambda)$.

\section{$6 \quad$ Numerical results}

In this section, we propose to validate the method on a linear problem: a uniform quasineutral stationary solution of the Vlasov-Poisson equation is perturbed. We then initialize 
the Vlasov-Poisson equation with

$$
f_{0}(x, v)=\frac{1}{\sqrt{2 \pi}}(1+\alpha \sin (\kappa x)) \exp \left(-\frac{v^{2}}{2}\right),
$$

on the interval $[0,2 \pi / \kappa]$, with periodic boundary conditions in the space direction and homogeneous Dirichlet boundary conditions in the velocity direction. As pointed out in section 2, the total energy is preserved with time at the continuous level. As a diagnostic, we then are interested in the time evolution of the kinetic, electrostatic and total energies $\mathcal{E}_{k}, \mathcal{E}_{p}$ and $\mathcal{E}_{t}$, respectively given by

$$
\mathcal{E}_{k}=\frac{1}{2} \iint f v^{2} d v d x, \quad \mathcal{E}_{p}=\frac{\lambda^{2}}{2} \int E^{2} d x, \quad \mathcal{E}_{t}=\mathcal{E}_{p}+\mathcal{E}_{k} .
$$

We also plot the electric field and the logarithm of the electric energy to accurately study the damping coefficient computed in the previous section. The same numerical test case has been studied in [9] using a PIC solver of the Vlasov equation coupled with the Reformulated Poisson Equation.

The numerical parameters are the following: $v_{\max }=6$ where the velocity domain extend from $-v_{\max }$ to $v_{\max }$, we use a number of cells $N_{v}=128$; the $\kappa$ parameter is taken equal to $\kappa=1, \alpha<<1$ to consider linear regimes, and $\Delta t=0.5 \Delta x / v_{\max }$.

The two different methods we detailed in section 3.1 are compared: the classical method uses the Ampère equation to predict the electric potential at time $t^{n+1}$ whereas the asymptotic stable approach uses the RPE discretization (3.16).

The initialization of the RPE scheme is done in the following way: we first compute the initial density $\rho^{0}$ thanks to the initial data $f^{0}$ and we assume that $\rho^{-1}=\rho^{0}$. Thanks to (3.16), we are able to compute $\phi^{1}$, the approximation of $\phi$ at time $\Delta t$. For the Ampère approach, classically the initial density $\rho^{0}$ enables us to compute $\phi^{0}$ according to the Poisson equation; then thanks to the initial current, we can advance the discrete Ampère equation (3.11) to get $\phi$ at time $\Delta t$.

On Figs. 3, 4 and 5, we give the results obtained by the two approaches with $\lambda=1$ and $\Delta x=2.4 \times 10^{-2}$, which results to a resolved case since $(\Delta x, \Delta t)<\lambda$. The kinetic, electric and total energies are plotted on Fig. 3, the electric field at time $t=2 \omega_{p}^{-1}$ and $t=10 \omega_{p}^{-1}$ is plotted on Fig. 4 and the logarithm of the electric energy on Fig. 5. For both methods, the results are stable since the stability constraint is fullfilled for the Ampère approach. The total energy is particularly well conserved with time for both methods. We can also observe that the results of the RPE approach are very close to the standard one on the different quantities we plot. Moreover, the numerical damping coefficient is in well agreement with that computed in the previous section for the two approaches. This test validates the RPE method with respect to the standard one.

On Figs. 6, 7 and 8, the same numerical parameters are considered but $\lambda=0.1$. The same conclusions as before are available for these results: both methods give accurate results with respect to the total energy conservation and to the damping coefficient. Moreover, the associated period of the wave is very close to the computed complex solution 
of the dispersion relation $(\omega=10.15-i 0.12$ whereas numerically we obtain $\omega=10.2-$ $i 0.1$ ).

Finally, Figs. 9, 10 and 11 present some results where $\lambda=10^{-2}$. In this case, the stability condition is not (strictly) respected in the standard approach neither in the RPE algorithm. However, both methods give stable results even if we can observe some differences. Indeed, on Fig. 10, the Ampère approach makes appear some oscillations on the electric field whereas the RPE one does not. The RPE method smoothes the microscale oscillations and consequently gives stable results, even when $\lambda<\Delta x$. On Fig. 11 the logarithm of the electric energy is plotted as a function of time. Up to $t \approx 4 \omega_{p}^{-1}$, both methods are nearly superimposed. First, the two curves present a highly damped behavior since the damping coefficient equals -10; then, a second behavior appears with a lower damping coefficient (about -1.73). We verify that these two behaviors are solutions to the dispersion relation; as mentionned in the section 4, the relation dispersion has several solutions, and two of them are captured by the numerical methods. From a quantitative point of view, the numerical methods are able to recover accurately the solutions of tables 1 and 2. For large times, the Ampère method seems to degenerate whereas the RPE approach appears to be more robust (we can observe a recurrence effect for example).

The last figures present numerical results for the RPE approach only. Indeed, when $\lambda=10^{-3}$ or $10^{-4}$, the Ampère approach gives rise to unstable results: the electric field generated by the Ampère equation becomes very strong which pushes the particles outside the velocity domain, so the total mass falls to zero. On Fig. 12, we observe that the total energy is still well preserved with time even if a decay occurs at the beginning of the simulation. This remains quite reasonable compared to the decay observed for PIC simulations in [9] due to the large noise resulting from the PIC assignment procedure. The use of a phase space grid solver seems to efficiently avoid this kind of phenomenom. On Fig. 14 the logarithm of the electric field also presents two different behaviors. They are both in a good agreement with the solutions of the dispersion relation we determined in section 4. On the contrary, in the Ampère context, since there exists one solution of the dispersion relation which gives rise to a positive imaginary part, the method leads to unstable numerical results.

Finally, the asymptotic preserving property is investigated considering very small values of $\lambda\left(\lambda=10^{-4}, 10^{-8}\right)$. We want to check if the numerical scheme tends towards a numerical approximation of the limit sytem of the Vlasov-Poisson system as $\lambda$ goes to zero. To that purpose, we compare our numerical results in which $\lambda=10^{-8}$ with the limit system (2.9)-(2.10). The numerical parameters are the same as previously. The initial condition with $\alpha=0$ has to be considered to respect the quasi-neutrality condition $\rho=1$ initially. In this case, the electric field is null everywhere and the Maxwellian initial condition is then a stationary solution. We can observe that the RPE method gives satisfactorying results since the electric field is very close to zero (see Fig. 16), and the total energy is equal to $\pi$ for large times. Fig. 18 shows that the total mass is equal to one for the RPE method whereas the total mass associated to the Ampère approach fails to zero, due to numerical instabilities. We can observe that for $\lambda$ close to zero, the RPE 


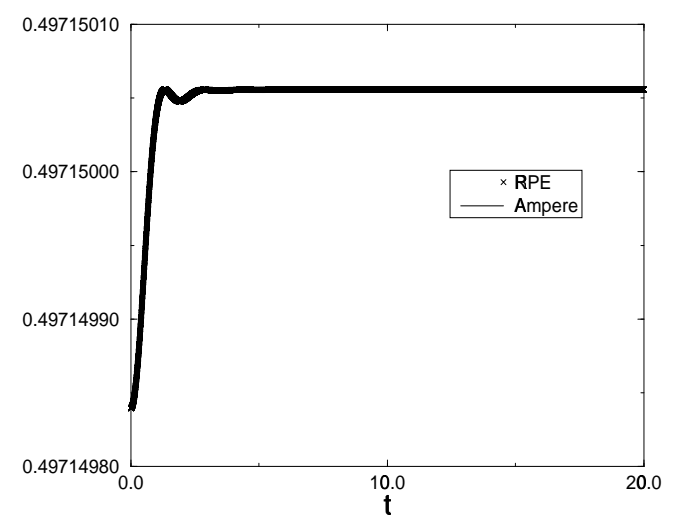

(a)

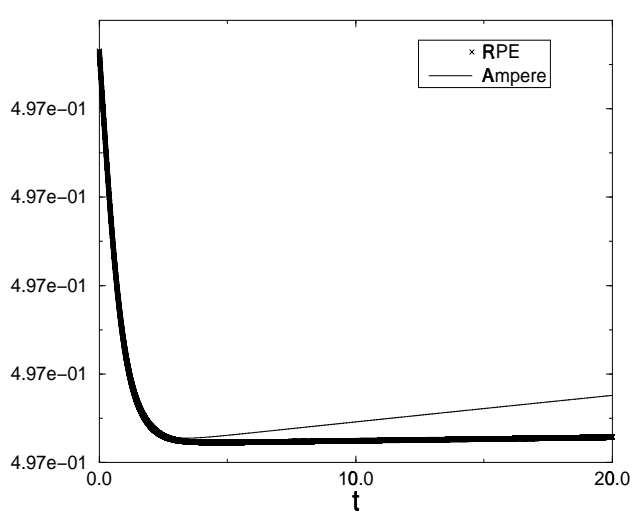

(b)

Figure 3: Comparison of the two methods: $\log \left(\mathcal{E}_{k}\right)$ as a function of time (left pannel), $\log \left(\mathcal{E}_{t}\right)$ as a function of time (right pannel). $\Delta x=2.10^{-2}, \quad \lambda=1$.

approach is able to numerically recover the quasi-neutral limit with a fixed grid of the phase space, i.e without resolving the small scales as the Debye length for example.

\section{Conclusion}

In this paper, we used a semi-Lagrangian scheme to simulate quasi-neutral problems using the kinetic description. In order to overcome the drastic stability condition $\lambda<\Delta x$, following [9], a Reformulated Poisson equation coupled with an appropriate time discretization of the characteristics curves has been implemented. An asymptotic preserving numerical scheme is then obtained, which enables to simulate quasi-neutral regimes. The present strategy has a comparable cost to that of an standard discretization.

\section{Appendix: Details for the computation of the com- plex integrals}

For the Ampère or the RPE case, we are led to compute integrals of the form

$$
\int \frac{\partial_{v} f_{0}}{v-\alpha} d v, \quad \alpha \in \mathbb{C},
$$

where $f_{0}$ is a Maxwellian

$$
f_{0}(v)=\frac{1}{\sqrt{2 \pi}} \exp \left(-v^{2} / 2\right) .
$$




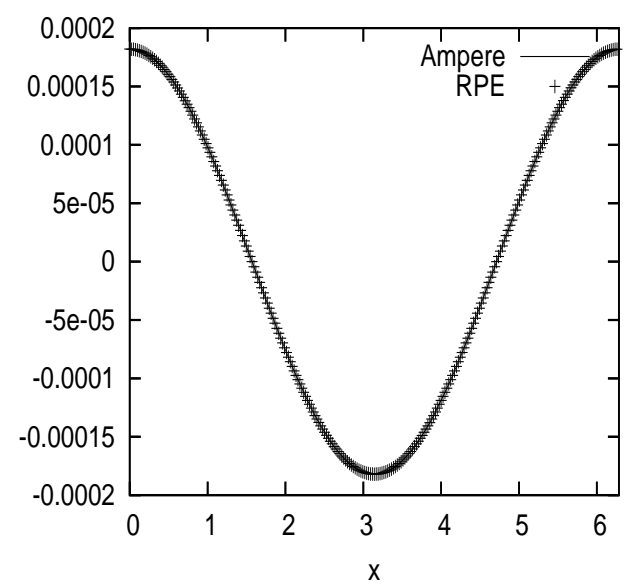

(a)

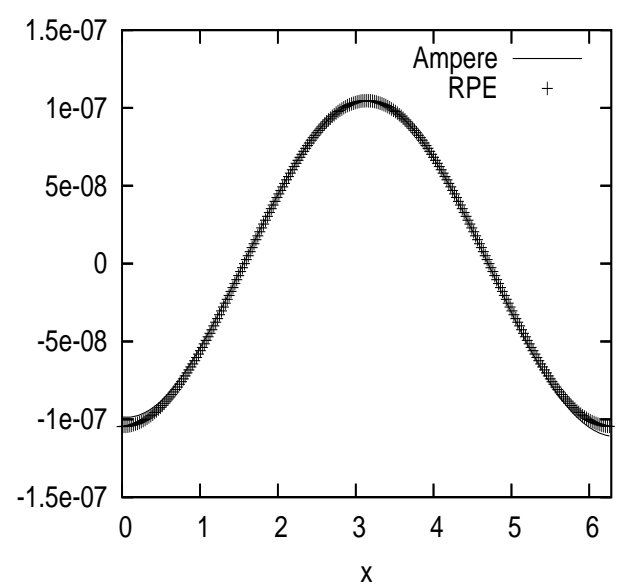

(b)

Figure 4: Comparison of the two methods: electric field as a function of the space variable at $t=2 \omega_{p}^{-1}$ (left pannel), and at $t=10 \omega_{p}^{-1}$ (right pannel). $\Delta x=2.10^{-2}, \lambda=1$.

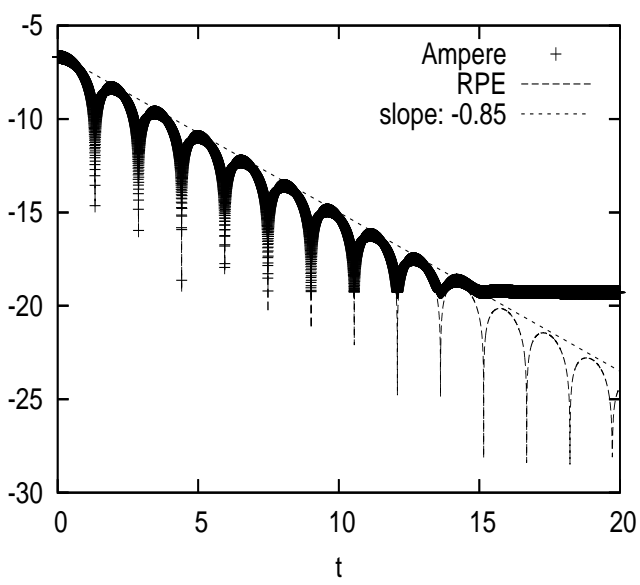

Figure 5: Comparison of the two methods: time evolution of $\log \left(\|E\|_{L^{2}}\right) . \quad \Delta x=$ $2.10^{-2}, \quad \lambda=1$. The slope -0.85 corresponds to the numerical Landau damping rate. 


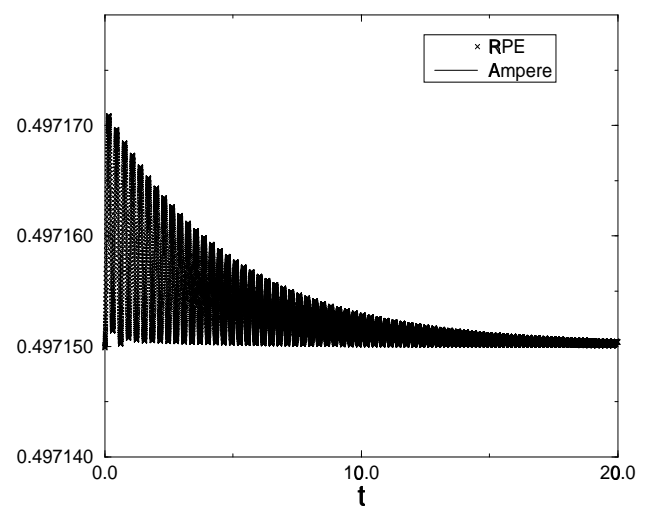

(a)

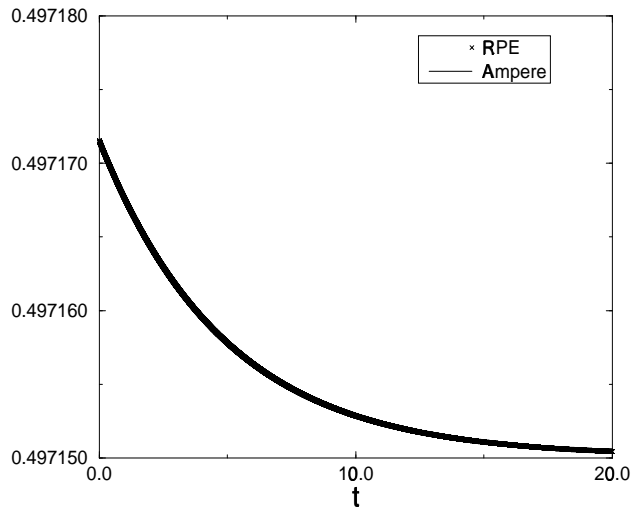

(b)

Figure 6: Comparison of the two methods: $\log \left(\mathcal{E}_{k}\right)$ as a function of time (left pannel), $\log \left(\mathcal{E}_{t}\right)$ as a function of time (right pannel). $\Delta x=2.10^{-2}, \quad \lambda=0.1$.

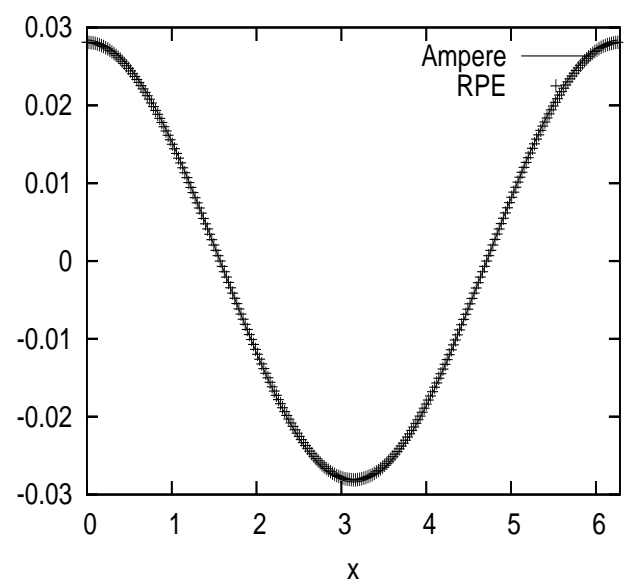

(a)

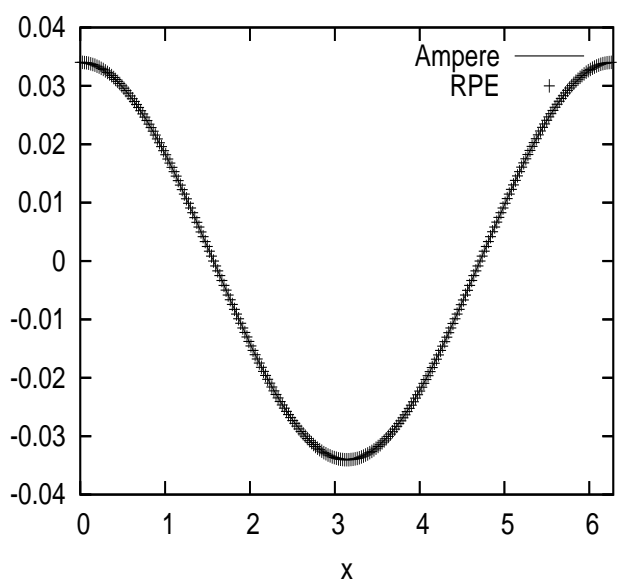

(b)

Figure 7: Comparison of the two methods: electric field as a function of the space variable at $t=2 \omega_{p}^{-1}$ (left pannel), and at $t=10 \omega_{p}^{-1}$ (right pannel). $\Delta x=2.10^{-2}, \quad \lambda=0.1$. 


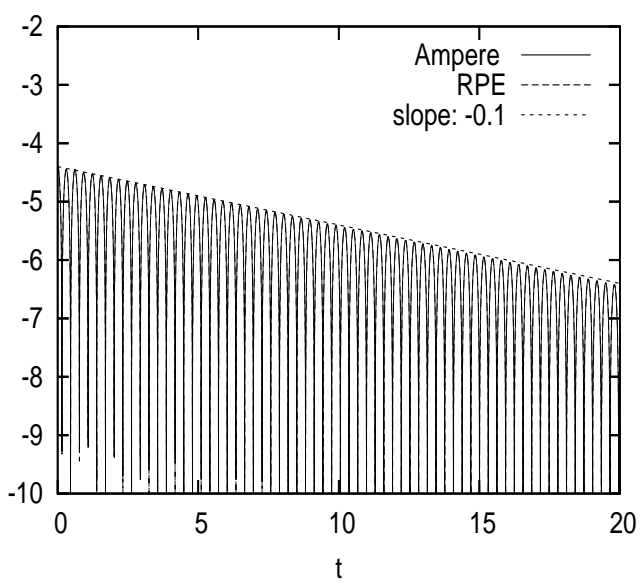

Figure 8: Comparison of the two methods: time evolution of $\log \left(\|E\|_{L^{2}}\right) . \quad \Delta x=$ $2.10^{-2}, \lambda=0.1$. The slope -0.1 corresponds to the numerical Landau damping rate.

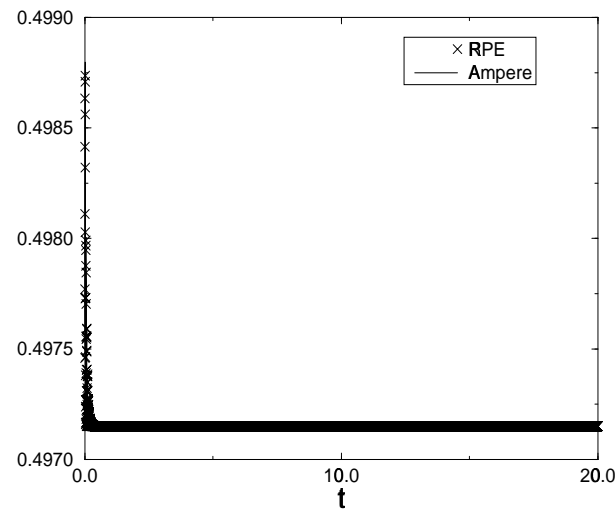

(a)

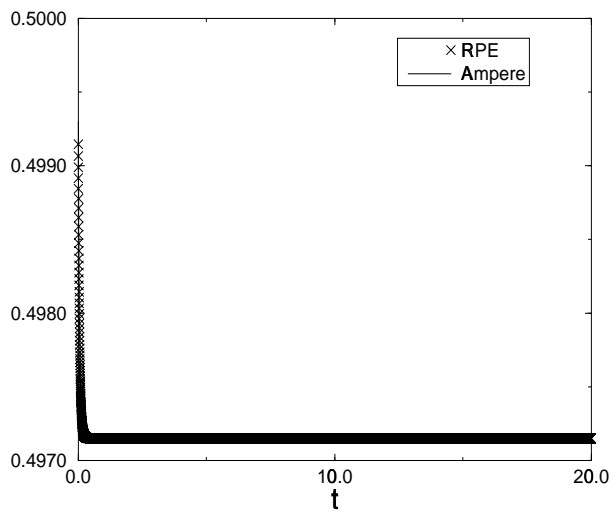

(b)

Figure 9: Comparison of the two methods: $\log \left(\mathcal{E}_{k}\right)$ as a function of time (left pannel), $\log \left(\mathcal{E}_{t}\right)$ as a function of time (right pannel). $\Delta x=2.10^{-2}, \quad \lambda=0.01$. 


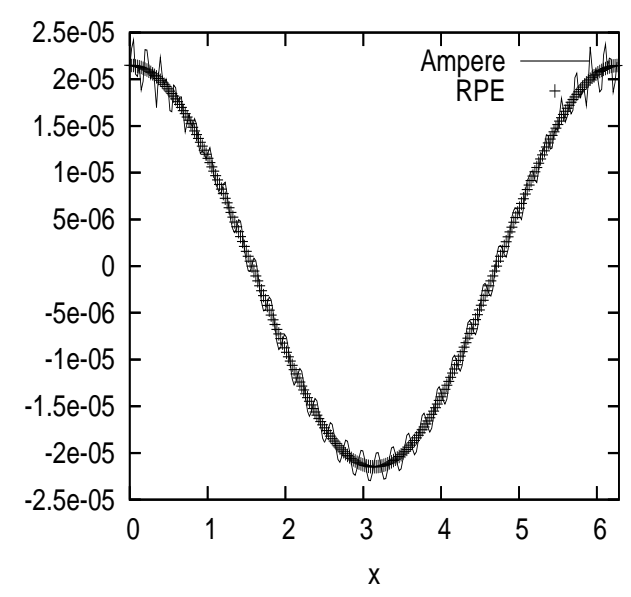

(a)

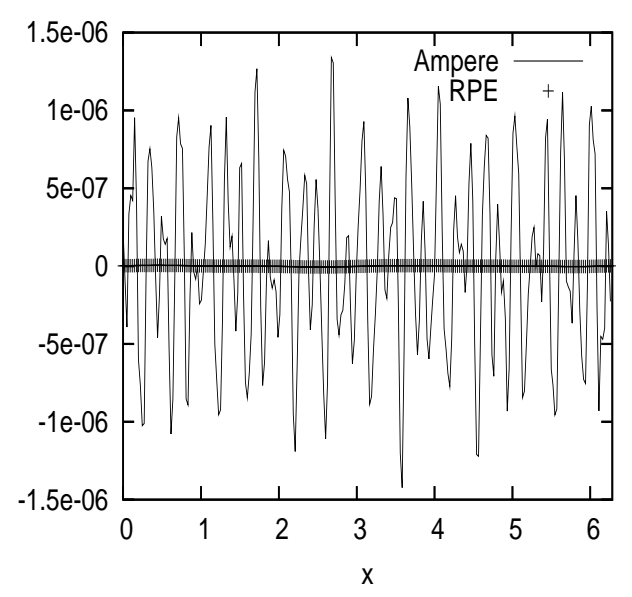

(b)

Figure 10: Comparison of the two methods: electric field as a function of the space variable at $t=2 \omega_{p}^{-1}$ (left pannel), and at $t=10 \omega_{p}^{-1}$ (right pannel). $\Delta x=2.10^{-2}, \lambda=0.01$.

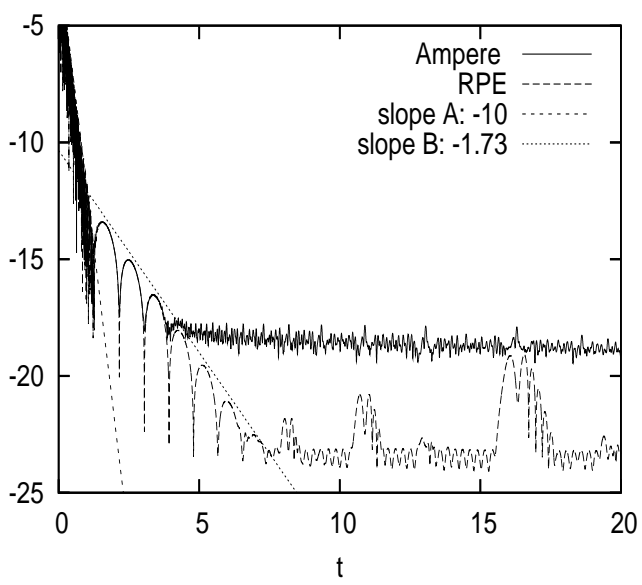

Figure 11: Comparison of the two methods: time evolution of $\log \left(\|E\|_{L^{2}}\right) . \quad \Delta x=$ $2.10^{-2}, \quad \lambda=0.01$. The slope $\mathrm{A}-10$ and the slope $\mathrm{B}-1.73$ correspond to the numerical Landau damping rates. 


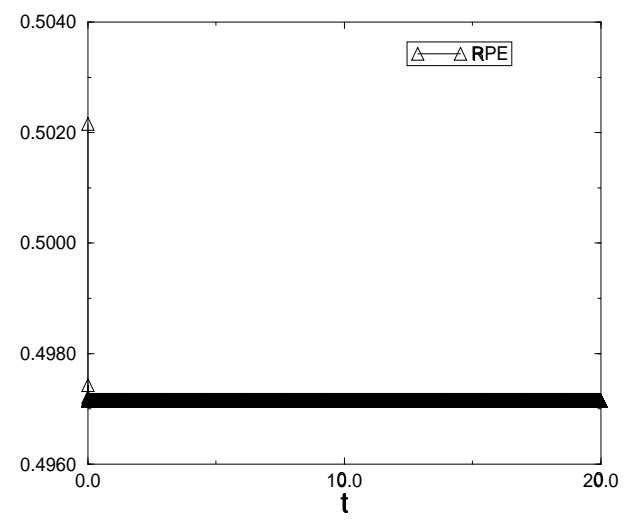

(a)

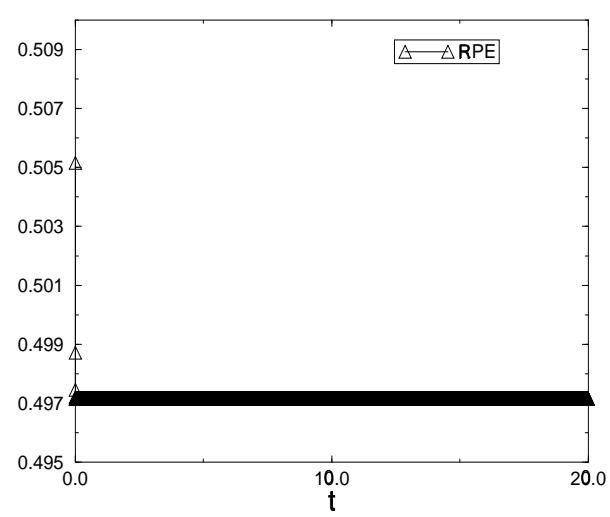

(b)

Figure 12: Numerical results for the RPE approach: $\log \left(\mathcal{E}_{k}\right)$ as a function of time (left pannel) $\log \left(\mathcal{E}_{t}\right)$ as a function of time (right pannel). $\Delta x=2.10^{-2}, \quad \lambda=0.001$.

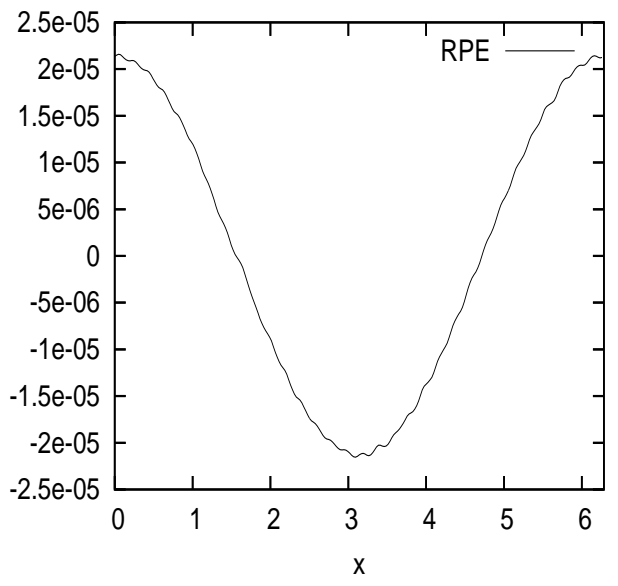

(a)

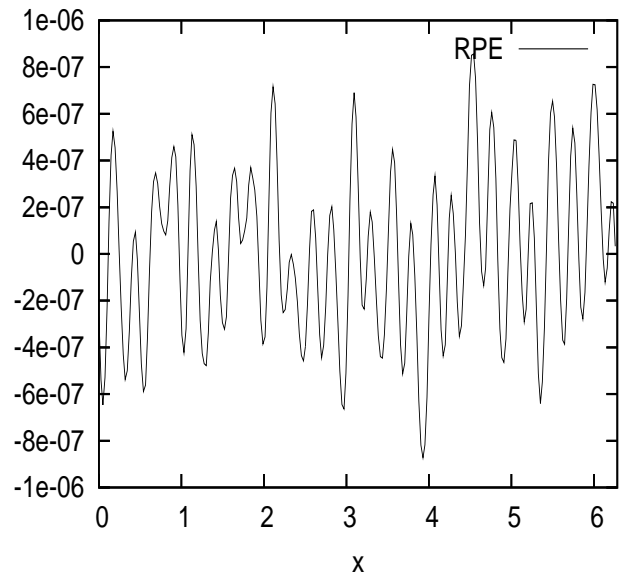

(b)

Figure 13: Numerical results for the RPE approach: electric field as a function of the space variable at $t=2 \omega_{p}^{-1}$ (left pannel), and at $t=10 \omega_{p}^{-1}$ (right pannel). $\Delta x=$ $2.10^{-2}, \quad \lambda=0.001$. 


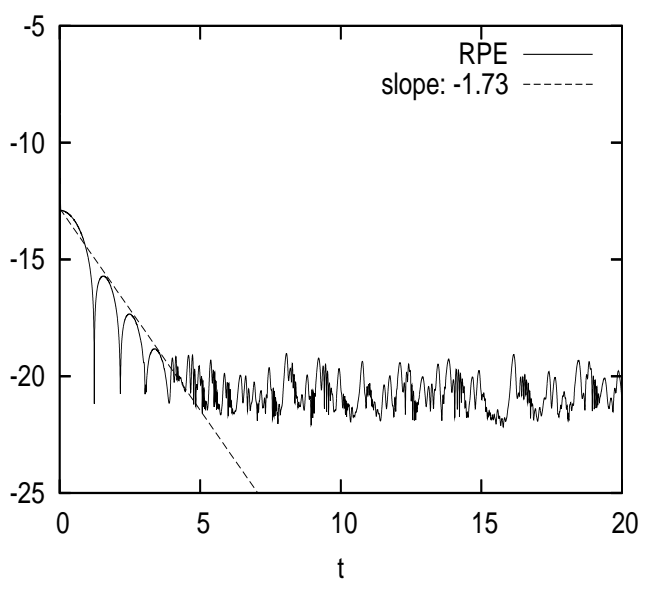

Figure 14: Numerical results for the RPE approach: time evolution of $\log \left(\|E\|_{L^{2}}\right) . \Delta x=$ $2.10^{-2}, \quad \lambda=0.001$. The slope -1.73 corresponds to the numerical Landau damping rate.

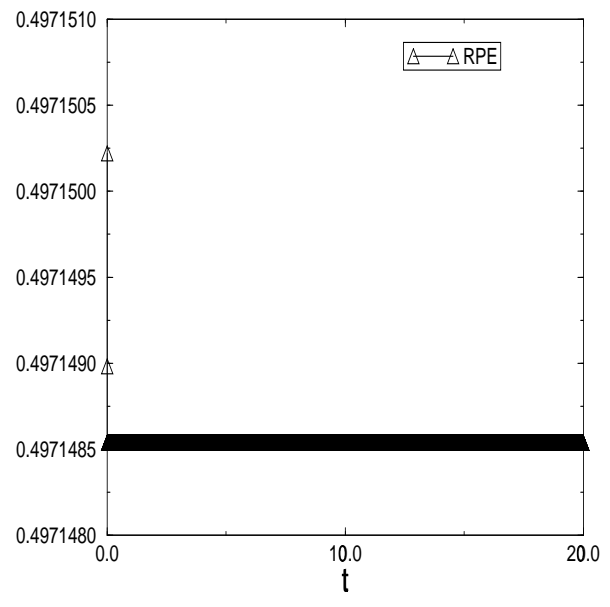

(a)

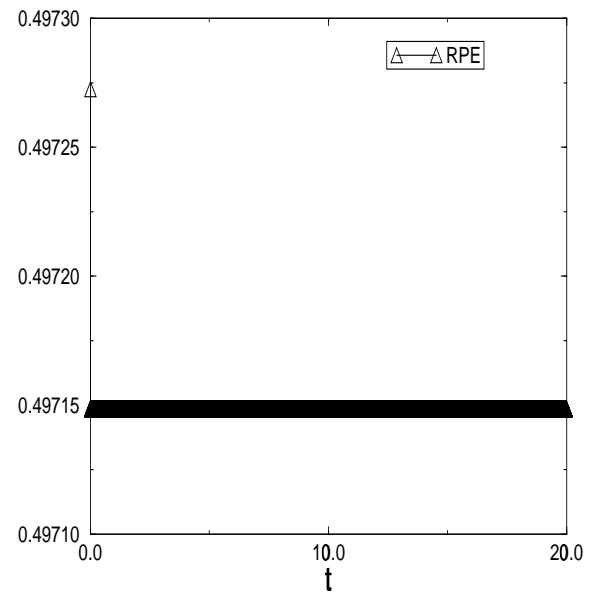

(b)

Figure 15: Numerical results for the RPE approach: $\log \left(\mathcal{E}_{k}\right)$ as a function of time (left pannel) $\log \left(\mathcal{E}_{t}\right)$ as a function of time (right pannel). $\Delta x=2.10^{-2}, \quad \lambda=0.0001$. 


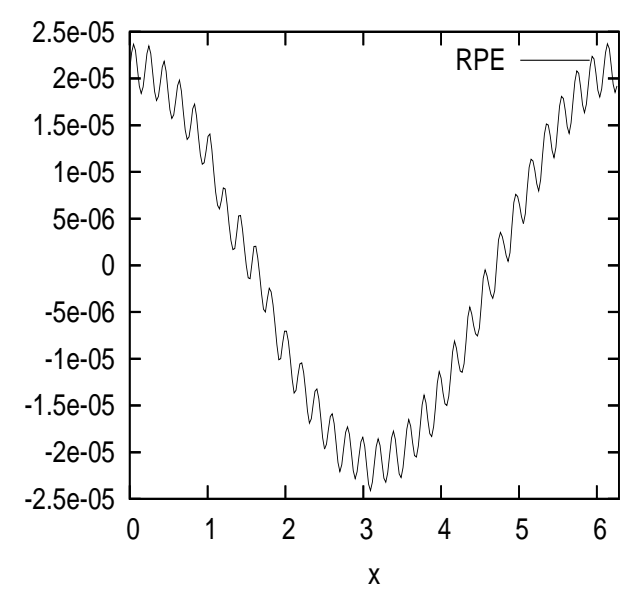

(a)

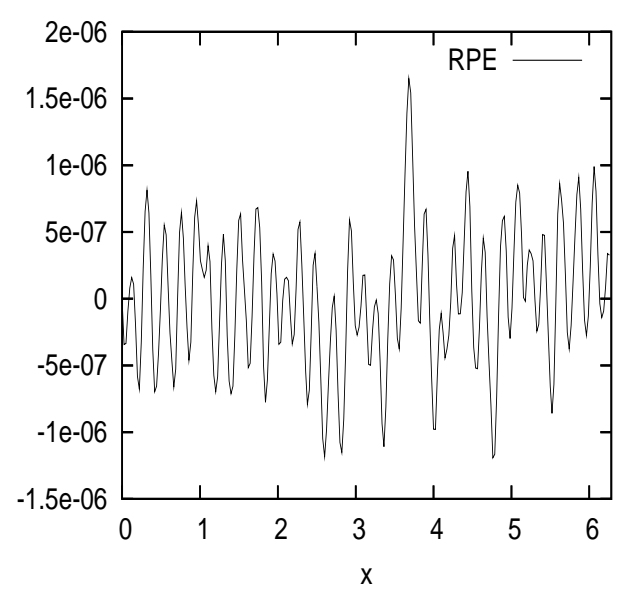

(b)

Figure 16: Numerical results for the RPE approach: electric field as a function of the space variable at $t=2 \omega_{p}^{-1}$ (left pannel), and at $t=10 \omega_{p}^{-1}$ (right pannel). $\Delta x=$ $2.10^{-2}, \quad \lambda=0.0001$.

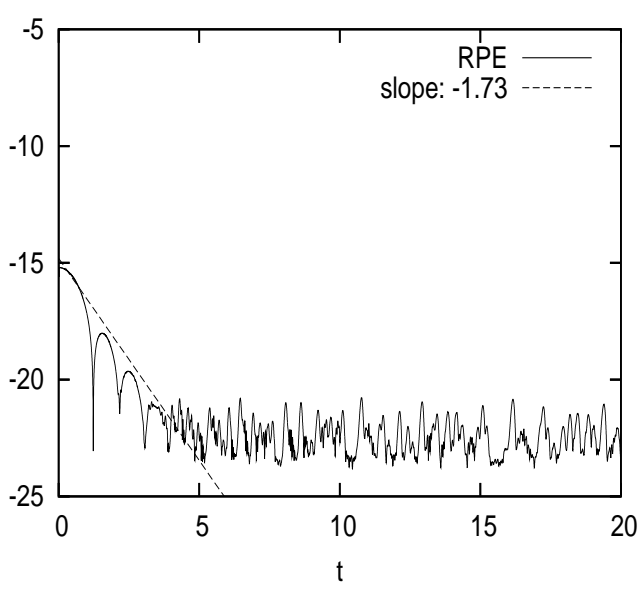

Figure 17: Numerical results for the RPE approach: time evolution of $\log \left(\|E\|_{L^{2}}\right) . \Delta x=$ $2.10^{-2}, \quad \lambda=0.0001$. The slope -1.73 corresponds to the numerical Landau damping rate. 


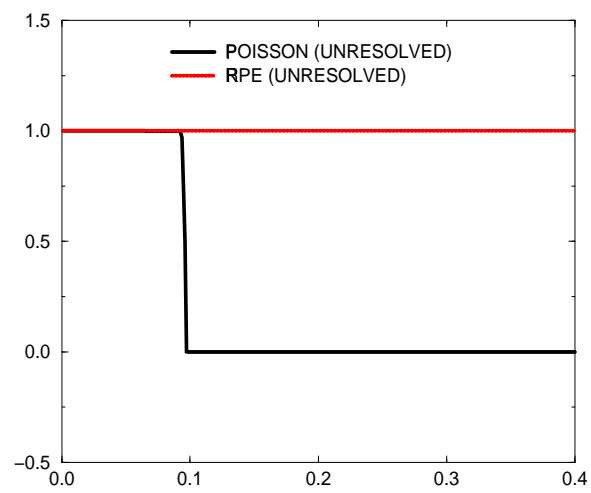

(a)

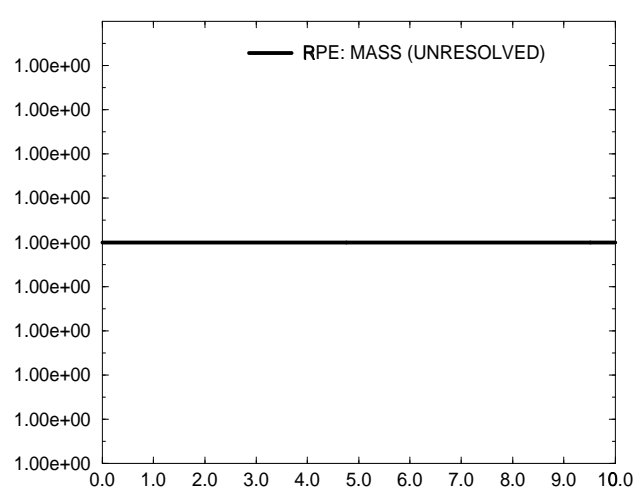

(b)

Figure 18: Comparison of the two methods: total mass as a function of time for $\lambda=10^{-4}$ (left pannel), and for $\lambda=10^{-8}$ (right pannel). $\Delta x=2 \cdot 10^{-2}$

Let us detail the computation in the following. Using an appropriate contour in the complex plane, we set

$$
\int \frac{\partial_{v} f_{0}}{v-\alpha} d v=\operatorname{Pr} \int \frac{\partial_{v} f_{0}(v+\alpha)}{v} d v+i \pi \partial_{v} f_{0}(\alpha)
$$

where the Cauchy principal value denoted by Pr is defined by

$$
\operatorname{Pr} \int_{-\infty}^{+\infty} \frac{g(v)}{v} d v=\lim _{\delta \rightarrow 0}\left[\int_{-\infty}^{-\delta} \frac{g(v)}{v} d v+\int_{\delta}^{+\infty} \frac{g(v)}{v} d v\right] .
$$

Let us detail the computations associated to the first term which we call $I$.

$$
\begin{aligned}
I & =-\frac{1}{\sqrt{2 \pi}} \operatorname{Pr} \int \frac{(v+\alpha) \exp \left(-(v+\alpha)^{2} / 2\right)}{v} d v \\
& =-\frac{1}{\sqrt{2 \pi}}\left[\int \exp \left(-(v+\alpha)^{2} / 2\right) d v+\alpha \operatorname{Pr} \int \frac{\exp \left(-(v+\alpha)^{2} / 2\right)}{v} d v\right] .
\end{aligned}
$$

Let us call $J$ the last term.

$$
\begin{aligned}
J & =\operatorname{Pr} \int \frac{\exp \left(-(v+\alpha)^{2} / 2\right)}{v} d v \\
& =\lim _{\delta \rightarrow 0}\left[\int_{-\infty}^{-\delta} \frac{\exp \left(-(v+\alpha)^{2} / 2\right)}{v} d v+\int_{\delta}^{+\infty} \frac{\exp \left(-(v+\alpha)^{2} / 2\right)}{v} d v\right] \\
& =\lim _{\delta \rightarrow 0}\left[\int_{\delta}^{+\infty} \exp \left(-\alpha^{2} / 2\right) \exp \left(-v^{2} / 2\right)(-\exp (\alpha v)+-\exp (-\alpha v)) d v\right] \\
& =-2 \exp \left(-\alpha^{2} / 2\right) \int_{0}^{+\infty} \exp \left(-v^{2} / 2\right) \operatorname{sh}(v \alpha) \frac{d v}{v}
\end{aligned}
$$


It is possible to express this last integral as a function of the erfi function, erfi $(x)=$ $(2 / \sqrt{\pi}) \int_{0}^{x} \exp \left(t^{2}\right) d t$. Indeed, noticing that

$$
y(x)=\int_{0}^{+\infty} \exp \left(-v^{2} / 2\right) \operatorname{sh}(v x) \frac{d v}{v},
$$

satisfies the differential equation $y^{\prime \prime}-x y^{\prime}=0$, we get that

$$
\int_{0}^{+\infty} \exp \left(-v^{2} / 2\right) \operatorname{sh}(v \alpha) \frac{d v}{v}=\frac{\pi}{2} \operatorname{erfi}\left(\frac{\alpha}{\sqrt{2}}\right) .
$$

Hence, gathering the previous terms leads to an expression of $I$

$$
I=-\frac{1}{\sqrt{2 \pi}}\left[\sqrt{2 \pi}-\alpha \exp \left(-\alpha^{2} / 2\right) \pi \operatorname{erfi}(\alpha / \sqrt{2})\right] .
$$

Finally, the initial complex integral we look for becomes

$$
\begin{aligned}
\int \frac{\partial_{v} f_{0}}{v-\alpha} d v & =-\frac{1}{\sqrt{2 \pi}}\left[\sqrt{2 \pi}-\alpha \exp \left(-\alpha^{2} / 2\right) \pi \operatorname{erfi}(\alpha / \sqrt{2})\right]-i \sqrt{\frac{\pi}{2}} \alpha \exp \left(-\alpha^{2} / 2\right) \\
& =-1+\alpha \sqrt{\frac{\pi}{2}} \exp \left(-\alpha^{2} / 2\right) \operatorname{erfi}(\alpha / \sqrt{2})-i \sqrt{\frac{\pi}{2}} \alpha \exp \left(-\alpha^{2} / 2\right) \\
& =-1+\alpha \sqrt{\frac{\pi}{2}} \exp \left(-\alpha^{2} / 2\right)(\operatorname{erfi}(\alpha / \sqrt{2})-i)
\end{aligned}
$$




\section{References}

[1] C.K. Birdsall, A.B. Langdon, Plasma Physics via Computer Simulation, Institute of Physics Publishing, Bristol and Philadelphia, 1991.

[2] Y. Brenier, Convergence of the Vlasov-Poisson system to the incompressible Euler equations, Comm. in Part. Diff. Eq., 25, pp. 737-754, (2000).

[3] C.Z. Cheng, G. Knorr, The integration of the Vlasov equation in configuration space, J. Comput. Phys., 22, p. 330, (1976).

[4] B.I. Cohen, A.B. Langdon, A. Friedman, Implicit time integration for plasma simulations, J. Comput. Phys. 46, p. 15, (1982).

[5] P. Crispel, P. Degond, M.-H. Vignal, An asymptotically stable discretization for the EulerPoisson system in the quasineutral limit, C. R. Acad. Sci. Paris, Ser. I 341 (2005) 341-346.

[6] P. Crispel, P. Degond, M.-H. Vignal, An asymptotically preserving scheme for the two-fluid Euler-Poisson model in the quasineutral limit, J. Comput. Phys., 203, pp. 208-234, (2007).

[7] N. Crouseilles, F. Filbet, Numerical approximation of collisional plasma by high order methods, J. Comput. Phys., 201, Issue 2, (2004), pp. 546-572.

[8] N. Crouseilles, G. Latu, E. Sonnendrücker, Hermite spline interpolation on patches for parallelly solving the Vlasov-Poisson equation, Int. J. Appl. Math. Comput. Sc., 2007, Vol. 17, No 3, pp. 101-115.

[9] P. Degond, F. Deluzet, L. Navoret, An asymptotically stable Particle-In-cell (PIC) scheme for collisionless plasma simulations near quasineutrality, C. R. Acad. Sci. Paris, Ser. I 343 (2006) 613-618.

[10] J.-L. Delcroix and A. Bers, Physique des plasmas 1, 2. Inter Editions-Editions du CNRS (1994).

[11] P. Degond, J.G. Liu, M.-H. Vignal, Analysis of an asymptotic preserving scheme for the Euler-Poisson system in the quasineutral limit, to appear in SIAM Numerical Analysis.

[12] P. Degond, C. Parzani, M.-H. Vignal, Plasma expansion in vacuum: modeling the breakdown of quasineutrality, SIAM Multiscale Modeling and Simulation 2, 158, (2003).

[13] S. Fabre Stability analysis of the Euler-Poisson equations, J. Comput. Phys., 101, 445 (1992).

[14] F. Filbet, E. Sonnendrǘcker, P. Bertrand, Conservative numerical schemes for the Vlasov equation, J. Comput. Phys., 172, pp. 166-187, (2001).

[15] F. Filbet, E. Sonnendrücker, Comparison of Eulerian Vlasov solvers, Comput. Phys. Comm., 151, pp. 247-266, (2003).

[16] B. D. Friede, S. D. Comte, The plasma dispersion function, Academic Press, New York, 1961.

[17] D.W. Hewett, C.W. Nielson, A multidimensional quasineutral plasma simulation model, J. Comput. Phys. 72, 121 (1987).

[18] R. W. Hockney, J.W. Eastwood, Computer Simulation Using Particles, Institue of physics, 1998. 
[19] N.A. Krall, A.W. Trivelpiece, Principles of plasma physics, San Francisco Press, 1996.

[20] A.B. Langdon, B.I. Cohen, A. Friedman, Direct implicit large time-step particle simulation of plasmas, J. Comput. Phys. 51, p. 107, (1983).

[21] A. Mankofsky, R.N. Sudan, J. Denavit, Hybrid simulation of ion beams in background plasma, J. Comput. Phys. 51, 484 (1983).

[22] R.J. Masson, Implicit moment particle simulations of plasmas, J. Comput. Phys. 41, p. 233, (1981).

[23] R.J. Masson, Implicit moment PIC-hybrid simulation of collisional plasmas, J. Comput. Phys. 51, p. 484, (1983).

[24] P.W. Rambo, Finite-grid instability in quasineutral hyrbid simulations, J. Comput. Phys. 118, $152(1995)$.

[25] S.Y. Ha, M. Slemrod, Global existence of plasma ion sheaths and their dynamics, Comm. Math. Phys. 238, 149 (2003).

[26] E. Sonnendrücker, J. Roche, P. Bertrand, A. Ghizzo, The semi-Lagrangian method for the numerical resolution of the Vlasov equations, J. Comput. Phys., 149, pp. 201-220, (1999).

[27] E Hairer, C. Lubich, G. Wanner, Geometric Numerical Integration, Structure-Preserving Algorithms for Ordinary Differential Equations, Series in Computational Mathematics, vol. 31, Springer, Berlin, 2002.

R. Belaouar : INRIA Nancy-Grand-EST (CALVI Project). and IRMA-Université Louis Pasteur, 7 rue René Descartes 67084 Strasbourg cedex, France.

belaouar@math.u-strasbg.fr

N. Crouseilles : INRIA Nancy-Grand-EST (CALVI Project), and IRMA-Université Louis Pasteur, 7 rue René Descartes 67084 Strasbourg cedex, France.

crouseil@math.u-strasbg.fr

P. Degond : Institut de Mathématiques de Toulouse, Université de Toulouse et CNRS (UMR 5219). Université Paul Sabatier, 118, route de Narbonne, 31062 Toulouse cedex, France.

degond@math. univ-toulouse.fr

E. Sonnendrücker : INRIA Nancy-Grand-Est (CALVI Project), and IRMA-Université Louis Pasteur, 7 rue René Descartes 67084 Strasbourg cedex, France.

sonnen@math.u-strasbg.fr 